\title{
Coupling from below as a source of ionospheric variability: a review
}

\author{
Edward S. Kazimirovsky \\ Institute of Solar-Terrestrial Physics, Russian Academy of Sciences, \\ Siberian Division, Irkutsk, Russia
}

\begin{abstract}
The purpose of this paper is to review shortly some observational backgrounds for the suggestion of a genuine link between processes in the lower atmosphere and ionospheric response. Attention is concentrated on the waves which are thought to couple the lower atmosphere with the thermosphere/ionosphere system.
\end{abstract}

Key words planetary waves - gravity waves middle-upper atmosphere interaction

«Every truth is fated to enjoy only one moment of triumph between the infinity when it is considered misleading, and the infinity when it is considered trivial...»

H. Poincare

\section{Introduction}

The study of the Earth's upper atmosphere is of interest to scientists from many disciplines and present day knowledge and understanding of the upper atmosphere to the highest levels is the result of the combined contributions of meteorologists, physicists, chemists, astronomers, geomagneticians, radio engineers and space scientists. This range of scientific disciplines has, not unexpectedly,

Mailing address: Prof. Edward S. Kazimirovsky, Institute of Solar-Terrestrial Physics, Russian Academy of Sciences, Siberian Division, Post Box 4026, 664033 Irkutsk 33, Russia; e-mail: ekazimirovsky@hotmail.com been matched by an equally extensive variety of experimental approach from standard meteorological instrumentation to the modern optical, radio and space-vehicle techniques yielding data from distances out to and far beyond, the limits of the atmosphere. These experimental studies have, over the years, been supplemented by much theoretical work to give the picture we now have of the physical state of the Earth's upper atmosphere. The terrestrial ionosphere is a part of the upper atmosphere, it is a cold magnetized plasma environment enveloping the Earth whose behaviour is often described as ionospheric aeronomy.

The Chambers Dictionary of Science and Technology denotes the term «aeronomy» as «the branch of science dealing with the atmosphere of the Earth and other planets with reference to their chemical composition, physical properties, relative motion and reaction to radiation from outer space». But the International Meteorological Glossary (1991) defines it more precisely: «A term sometimes used to denote that branch of the Earth's atmospheric physics which is concerned with those regions, upwards of about 50 $\mathrm{km}$, where dissociation and ionization are fundamental processes». 
The principal physical, chemical and electrical properties of the Earth's upper atmosphere are very largely the result of its interaction with solar wave and particle radiation. The ultraviolet and $\mathrm{X}$-radiations are absorbed at various levels between about 40 and $200 \mathrm{~km}$. The selective absorption of the ultraviolet and X-radiation by particular atmospheric constituents gives rise to the unique electrical properties of the upper atmosphere by providing a series of ionized strata (layers) collectively known as the ionosphere. Other ultraviolet radiation is very effectively absorbed lower down in the atmosphere by ozone.

Owing to the pervasive influence of gravity, the atmosphere and ionosphere are to first order horizontally stratified and conventionally divided into layers based on the vertical structure of different parameters. The atmospheric structure can be neatly organized by a representative temperature profile, while the ionosphere is more sensibly organised by the number density of plasma. A schematic representation of atmospheric regions is shown in fig. 1 (Whitten and Poppoff, 1971).

In the following text we use the terms «upper atmosphere» and «middle atmosphere», which are absent in fig. 1 . In the past, meteorologists often designated the entire region above the tropopause as the «upper atmosphere». But rather recently the term «middle atmosphere» became popular in referring to the region from the tropopause to the turbopause and even to the lower thermosphere.

In addition to electromagnetic (wave) radiations, the Sun also emits continuously streams of energetic electrically charged particles - mainly protons and electrons. This is the so-called «solar wind» - an outward flow of charged particles moving at velocities of a few hundred kilometers per second. They interact with the geomagnetic field in a complicated way, and with the gases of the upper atmosphere. As a result, we can observe the geomagnetic storms, the disturbances to long-distance radio-wave communication via the ionosphere and the visible auroral displays at high latitudes. All these events are closely associated with the occurrence of sunspots and show characteristic time variations over 11 years and 27 days, respectively, related to the sunspot cycle and the period of rotation of the Sun. The upper atmosphere/ionosphere acts as the intermediary between the plasma-dominated magnetosphere and the bulk of the neutral atmosphere below. This region is highly complex. Interacting dynamic, chemical, radiative and electrical variations occur there. To understand how these coupled elements interact to produce the great variability characteristic of the system is one of the major problems in solar-terrestrial relations. For example, the three-dimensional circulation of the thermosphere changes during and following geomagnetic storms; yet the consequences of the change in circulation on the temperature, density, composition and electric currents of the region are poorly understood. Energetic solar particles penetrate the middle atmosphere and produce chemical changes in radiatively important species such as ozone, but their global consequences are not fully appreciated. Deeper in the atmosphere, solar induced variations in the flux of cosmic rays may produce variations in the electrical structure of the lower atmosphere, but the effects of these variations on the Earth's global electrical circuit (including the ionosphere) are not fully understood.

The Earth's ionosphere is a partially ionized gas that envelops the Earth and in some sense forms the interface between the atmosphere and space. Since the gas is ionized it cannot be fully described by the equations of the neutral fluid dynamics. On the other hand, the number density of the neutral gas exceeds that of the ionospheric plasma and certainly neutral particles cannot be ignored. Therefore the knowledge only of two «pure» branches of physics: classical fluid dynamics and plasma physics is not sufficient. In addition to atmospheric dynamics, space physics, ion chemistry and photochemistry are necessary to understand how the ionosphere is formed and buffeted by sources from above and below and to deal with production and loss processes.

Until recently, the ionosphere was studied as a merely magneto-active plasma without consideration of the general properties of the atmosphere. It was known that the neutral atmosphere (thermosphere) and ionosphere are 


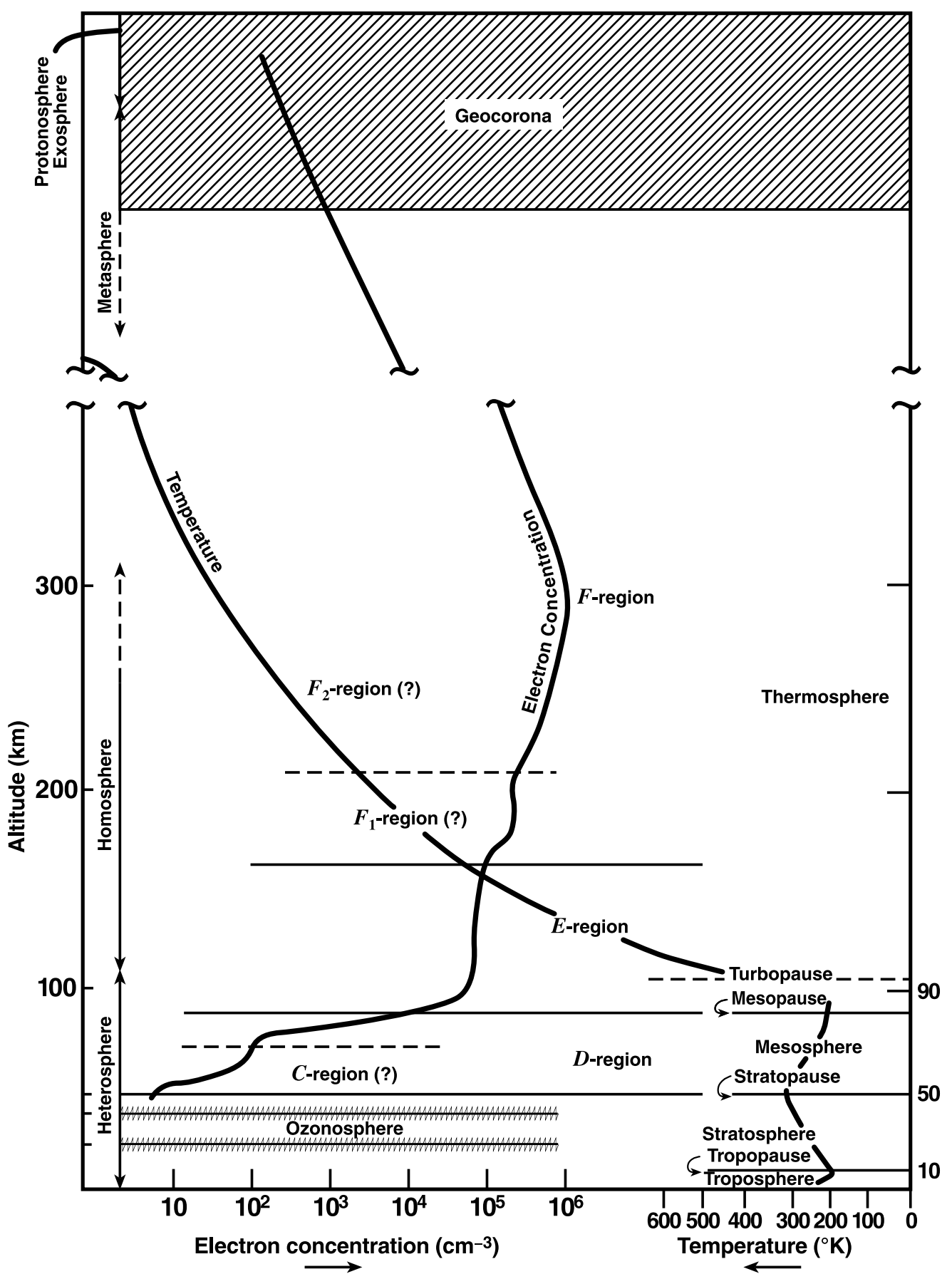

Fig. 1. Schematic representation of atmospheric regions. Classification of atmospheric layers in accordance with temperature (right) and with electron concentration (left) (Whitten and Popoff, 1971). 
linked by electrodynamic and momentum transfer. Nevertheless, almost nobody believed that the terms «meteorology» and «climatology» can be applied to the ionosphere, it was thought that no connection existed between events occurring in the troposphere/ stratosphere system on one hand, and in the ionosphere on the other. Meanwhile, however, it has become apparent that the composition, chemistry, energetics, dynamics and resulting structure of the lower and upper atmosphere, which are functions of location and time, are so intricately interrelated that it is not really possible to discuss each of them in isolation. Gradually, the viewpoint that the lower and upper atmosphere are substantially uncoupled was rejected. Many discoveries and experimental results have confirmed the existence of detailed correlations between the parameters of the lower and upper atmosphere (Kazimirovsky and Kokourov, 1991).

But what is the reason for the correlations? As part of a physical mechanism, the influence of internal atmospheric waves may be considered. The upward propagation of internal atmospheric waves (planetary waves, tides and gravity waves) from the troposphere and stratosphere is an essential source of energy and momentum for the thermosphere and ionosphere. Of course, the study of internal waves is the province of meteorology, a discipline that has enjoyed a long and independent development of its own and has its own complicated problems, sufficiently different from ionospheric physics that the two are regarded as separate but neighbouring disciplines. However, the internal waves launched by weather fronts or any other sources in the troposphere and stratosphere sometimes appear to be capable of penetrating into the ionosphere, where they dissipate their energy. The leakage of wave energy from the troposphere and stratosphere at least up to 100$115 \mathrm{~km}$ was introduced as «coupling from below» (Bowhill, 1969) and is considered as a mechanism of the meteorological influence on the ionosphere. This influence has been assumed and is presented by various models.

The meteorology of the thermosphere differs considerably from that associated with the familiar weather patterns we experience at the Earth surface, although the fluid motions are governed by the same equations as those used by meteorologists studying the weather systems. In the thermosphere, temperature increases with altitude, making for a dynamic system that is less dominated by instabilities than the troposphere, where the temperature gradient is in the opposite direction. Also, the viscous and ion drag forces are very important in the thermosphere, with the former tending to transfer momentum between various altitudes and the latter acting to strongly couple the neutral thermosphere to the ionosphere and thereby, to the magnetosphere. It is now established beyond doubt that the atmosphere extends from the ground to the thermosphere, behaving as a complex system coupling fairly closely over wide height ranges.

For the aeronomical models, the major questions now posed relate to the interactions or coupling between regions, interactions that are currently only crudely parameterized within the separate models. This is particularly true for the upper atmosphere, where a rich set of physical processes have been identified that couple the various regions together, as well as to the stratosphere and troposphere below and to the magnetosphere above. Therefore, the principal scientific challenge facing us today is to understand the coupled system as a whole, including the effects of energy, momentum and compositional interchange between regions.

The analysis of «traditional» meteorological and ionospheric data is not only of interest for the exploration of purely theoretical aspects of the stochastic atmosphere system, but also of relevance for modern climatological research. For instance, it is a still unsolved question as to how much the general state of the middle and thus the lower atmosphere and its circulation systems are influenced by changes with various timescales at the upper boundary - thermosphere/ ionosphere. Such changes may even be left in the troposphere. Since the upper atmosphere is generally a good indicator of solar activity, one might assume that the correlation between tropospheric and ionospheric parameters indicates such a solar-atmosphere or solarweather effect. Although it is easier to picture 
upward dynamic coupling because of the much greater energy density that resides in the lower atmosphere relative to that of the upper atmosphere, it is possible that changes in the upper atmosphere can give rise to significant changes in the lower atmosphere. The subject of solar activity effects on tropospheric weather is still controversial. Less controversial, however, is the possibility of solar activity effects on climate. But the very interesting problem «The role of the Sun in Climate Change» requires special consideration elsewhere.

Our main concept is that the science of the whole atmosphere-ionosphere system becomes greater than the sum of its component parts. The scientific focus is placed on the interactive processes among the various physical regimes. This concept is in the base of International SolarTerrestrial Energy Program (STEP) and postSTEP activity, comprehensive study of the mutual linkages between the various regions of space in addition to the traditional study of the individual regions themselves.

Many of the ionospheric phenomena cannot be explained only in terms of the ionizing radiation variations, photochemical processes, solar particle injections or solar flare effect. It seems likely that there are some events due to the effect of atmospheric oscillations, such as the winter anomaly of radiowave absorption, $E$-sporadic layer occurrence and structure, thermospheric wind regime, the variations of atmospheric emissions, travelling ionospheric disturbances, day-to-day midlatitude ionospheric variability, $F$-spread event, etc. Finally, the atmosphere and ionosphere are linked dynamically, radiatively and chemically. It is why in the following paragraphes we discuss the atmospheric dynamics and ionosphere from the meteorological positions. Aeronomy is now the study of the upper atmosphere physical and chemical processes including ionospheric processes. Electrical engineers are interested in the propagation of radiowaves; physicists in plasma processes; chemists in the dissociation and diffusion of atmospheric molecules and atoms; meteorologists in dynamics; and so on. As the body of knowledge grew, it became apparent that all aspects are important and that, in fact all processes are interdependent. In upper atmosphere research, as in no other field, the disciplines of electrical engineering, meteorology, chemistry and physics are intertwined (Whitten and Poppoff, 1971; Danilov et al., 1987; Hargreaves, 1992; Johnson and Killeen, 1995; Wickwar and Carlson, 1999; Solomon, 2000; Forbes et al., 2000 ).

The purpose of this paper is to review briefly some observational backgrounds for the suggestion of a genuine link between processes in the lower atmosphere and ionospheric response. Attention is concentrated on the waves which are thought to couple the lower atmosphere with the thermosphere/ionosphere system. Detailed explanations of the observations and theories are not provided. Of course, the survey presented here will be affected by the personal biases of the author and the limitations of space. Since the discussion is intended to be «tutorial» in nature, only a few key references to the literature will be given, and the interested reader can consult these for more detailed discussion and for links to the more extensive literature.

\section{Internal atmospheric waves}

One exciting aspect of modern ionospheric research is the significant role of the lower atmosphere in upper-atmospheric variability. The forcing could come from internal atmospheric waves (planetary waves, tides, internal gravity waves).

Planetary waves are a well-documented group of atmospheric waves, a class of zonally travelling structures of global scale, which have periods of a few days (typically 2-30 days). Such waves appear for example in surface pressure data, in standard analyses of upper tropospheric radiosonde data and in satellite stratospheric data.

A significant part of the energy of the planetary wave disturbances of the troposphere may propagate into the upper atmosphere and the effective index of refraction for the planetary waves depends primarily on the distribution of the mean zonal wind with height.

The International Reference Atmospheric Model (CIRA-1990) includes the description of planetary waves up to $85 \mathrm{~km}$ with the following general features: 
1) Wave amplitudes are small in the tropics throughout the year, slightly larger in the summer season at mid and high latitudes and much larger during the winter season.

2) Maximum amplitudes occur at about 60$70^{\circ}$ north or south latitude during local winter.

3) Amplitudes are generally largest in the stratosphere and lower mesosphere but they remain relatively large up to the top level lower thermosphere.

There is one important peculiarity in the theory of planetary wave propagation, associated with the existence of critical surfaces or layers, where the basic zonal wind matches the zonal phase speed of wave. The dissipation and nonlinearity become very important in this critical layer. It may be expected to be an enhanced dissipative region for planetary waves or under some conditions the critical layer remains a perfect reflector of planetary waves.

The most spectacular manifestations of planetary waves activity in the middle atmosphere, associated with strong coupling between the stratosphere and lower atmosphere, occur during stratospheric sudden warming events. The zonal-mean climatological temperature and zonal wind configuration is dramatically disrupted, with polar stratospheric temperatures increasing rapidly with time, leading on occasion to reversals of zonal-mean winds from westerlies to easterlies (Naujokat and Labitzke, 1993).

Current theories suggest that a major sudden warming is initiated by an anomalous growth of a planetary-wave disturbance (mainly comprising wave-number 1 and 2 components) that propagates from the troposphere into the stratosphere and interacts strongly with the preexisting circulation there. The stationary planetary waves that propagate energy upward also transport heat southward (Andrews et al., 1987).

Our understanding of all the observed details of these events, the necessary conditions for their occurrence, interannual variability between one stratospheric winter and another is still by no means complete. Much further work will need to be done before a full understanding of the phenomenon is attained.

In general, the implication of the theory for the modelling of planetary waves and for interpretation of atmospheric observations are not yet absolutely clear and new experimental information concerning the behaviour and effects of planetary waves, especially in the lower and upper thermosphere is very important. Since the seasonal and year-to-year variability of planetary wave activities cannot be simply explained by the steady-state propagation theory, the variations of forcing in the lower atmosphere would be important.

Atmospheric tides are global-scale oscillations, which are primarily forced by variations of heating due to absorption of solar ultraviolet radiations by atmospheric water vapour in the troposphere, ozone in the middle atmosphere and molecular oxygen $\mathrm{O}_{2}$ in the lower thermosphere. The solar and lunar gravitational forcing that produces ocean tides is much less important for the atmosphere.

The migrating tides (diurnal, semi-diurnal, etc.) can propagate through great depths of the atmosphere and can attain large amplitudes at some heights especially in the thermosphere. The semidiurnal tide plays a particularly important role in the lower thermosphere, where the global temperature and density variations are dominated by this mode. At higher altitudes the semi-diurnal tide is dissipated by viscosity and ion drag. In the upper thermosphere, at $300 \mathrm{~km}$, the amplitude of the semi-diurnal tide decreases, and thermospheric density variations are dominated by the diurnal tide that has been forced by the thermospheric absorption of EUV solar radiation. In the modern theoretical calculations the semidiurnal tide in the thermosphere is considered as a result of propagation from below.

The non-migrating tides (associated, for example, with orography and geographically fixed tropospheric heat sources) would give rise to longitudinal differences in tidal structure.

In the classical tidal theory (in viscid atmosphere, background temperature is independent of latitude) the governing equation is separable, giving rise to vertical and latitude structure equations. But in the real atmosphere with winds and meridional gradients of temperature the governing equation may be solved only numerically. 
Now the modellers try to include in the models the seasonal, latitudinal and longitudinal variations, realistic temperature and wind structures, molecular and eddy diffusion, acceleration and heating of the mean flow by tides, the effect of tides on minor constituent concentration, hydromagnetic coupling - all for viscid, rotating, spherical atmosphere (e.g., Forbes, 1991).

Internal Gravity Waves (IGW) are disturbances which are allowed to propagate as a consequence of buoyant forces present in the atmosphere. The temperature and wind structures determine the wave's propagation characteristics.

Many seasonally and latitudinally varying sources for middle atmosphere gravity waves have been identified. These include airflow over orography, severe weather fronts, cyclones, instabilities in the planetary boundary layer and in jet stream shears, turbulent motions of different scales, thunderstorms ( e.g., Vincent, 1990; Gavrilov, 1992).

At heights above $85-90 \mathrm{~km}$, the internal gravity waves may be «saturated» and even be broken with deposition of energy and momentum. The "trapping» of IGW is also possible, so that one would expect IGW to be ducted in the region near the mesopause. It is now believed that the level of gravity wave activity determines the mean state of the mesosphere. Moreover, in the lower thermosphere the waves manifest themselves in wind, temperature, density, pressure, ionization, vertical profiles of $\mathrm{Na}$, measured by lidars, and airflow fluctuations in the $80-120 \mathrm{~km}$ height range and the amplitudes are so large that they can dominate at these altitudes (e.g., Krassovsky, 1977; Vincent, 1990; Gavrilov, 1992).

It is now well appreciated that internal gravity waves play an important role in determining the mean circulation and thermal structure of the middle and upper atmosphere. There are theoretical models of the interaction between a gravity wave and the ionization oscillations of ionic velocity and temperature, influence on the electron density, effect on the electron temperature, generation of ionospheric irregularities, etc.

\section{The lower thermosphere/ionosphere}

An atmospheric layer located at about 60 to $100 \mathrm{~km}$ altitude can be defined as a transition region, where different fundamental physical mechanisms, dominant in the lower and upper height regions, coexist, showing complicated atmospheric characteristics. For instance, the $D$-region, the ionosphere at $60-90 \mathrm{~km}$, weakly ionized (mainly in daytime), is an interface between the neutral and ionized atmospheric layers. The $D$-region is the lowest lying ionospheric region and hence is produced by the most penetrating of the ionizing radiation (Galactic Cosmic Radiation, X-rays, the intense Solar hydrogen Lyman-alpha emission line and Extra-Ultra-Violet); it is a region of weaklyionized plasma and large neutral species numberdensity as well as complex ion-interchange and electron attachment and detachment reactions (the latter processes are the most distinguishing feature of the $D$-region). The perturbations of this region greatly affect the absorption of high frequency radio waves and the reflection of the low-frequency signals.

Various techniques are available for the exploration of the plasma density in the $D$-region. Much observational evidence has accumulated showing that its behaviour is far less simple and straightforward than the regular «Chapman-layerlike» behaviour controlled only by solar energy influx and the geometry of its penetration into the atmosphere. The diurnal and especially seasonal variations sometimes demonstrate the significant abnormal peculiarities especially in winter. At the same time groundbased, rocket and satellite-board soundings of temperatures and winds in the upper mesosphere-lower thermosphere (MLT-region) exhibit variation patterns with temporal and spatial scales which are to be described by the term «meteorology». Thus, nowadays it is possible to introduce into practice the «meteorological control» of the $D$-region for the explanations of some events (Taubenheim, 1983; Danilov et al.,1987). It means that the lower ionosphere exhibits not only a solar, but also a strong non-solar control which is partly of a meteorological nature.

The most suitable for the study of meteorological effects upon the $D$-region are groundbased 
radio propagation observations, because they provide data records continuous in time in addition to the singular rocket launches. The experimental basement for the intensive investigations of the coupling so far are the ionospheric radiowave absorption and dynamic regime (prevailing winds and wave-like fluctuations) measurements in the MLT region. The radiosignals reflected from or propagated through the ionosphere depend on the effect known as absorption, or attenuation of electromagnetic wave energy due to collision of ionospheric electrons with neutral atoms. Without considering the details of magnetoionic theory it could be summarized that absorption has a direct relationship with the product of plasma density and the collision frequency and an inverse relationship with the emitted radiowave frequency. The most investigated $D$-region absorption data are produced by the measurements known as A3 method described in all details by Schwentek (1976). That consists in measuring the attenuation of MF radiowaves radiated from a distant transmitter with constant power.

The best known manifestation of a meteorological influence upon the $D$-region is the winter anomaly, i.e. excessive enhancement of both the average level and especially day-to-day variability («spikes») of radio-wave absorption in winter, noted by many investigators even in the first years of ionospheric science. The example of winter anomaly in terms of absorption at a constant solar zenith angle during 19671969 is shown in fig. 2 (Taubenheim, 1983).

There are two components: 1) «normal» winter anomaly with a rather slow increase and decrease, i.e. in average daytime absorption for usual winter day is higher than for usual summer day; 2) superimposed spikes, «excessive» winter anomaly. Although enhancements of absorption may be connected with increasing electron density and/or collision frequency in ionospheric plasma, it is nowadays assumed that the winter anomaly is connected primarily with enhanced electron densities at the mesopause level and above.

Why do we assume that the winter enhancement of electron density has an internal («meteorological») nature?
1) It occurs on both the northern and the southern hemispheres during the respective winter months.

2) The correlation between the time variations of $D$-region ionization and solar $L$-alpha fluxes (measured by AE-E satellite), which is weak but detectable during summer months, is completely missing during winter.

3) The amplitude of interdiurnal variations of absorption in winter months cannot be explained by solar $L$-alpha variations.

4) The time and spatial scales of anomalous absorption, wave-like structures, are compatible with planetary waves pattern (e.g., de la Morena and Kazimirovsky, 1996).

There are some «scenarios», concerning the $D$-region meteorological control (e.g., Taubenheim, 1983; Offerman et al., 1982):

1) «Concerted» Scenario.

- The general enhancement of $D$-region electron densities is caused by enhanced downward eddy diffusion of NO, accumulated in the polar night thermosphere.

- In addition, at heights below $85 \mathrm{~km}$, the electron rate is reduced by inhibition of cluster ion formation because of warm mesopause temperatures.

- Independently, «patchy» (with respect to time and longitude) downward transports of excess NO into the $D$-region are effected by diffusion and/or bulk motions connected with planetary wave patterns. Displacement of these patches by enhanced horizontal winds, possibly launched by transient perturbations from below, causes rapid changes in the NO distribution within 1-2 days.

2) «Unitary» Scenario.

- Winter meteorology at $D$-region heights is predominantly controlled by the circumpolar vortex of zonal westerly winds, reaching from the stratosphere up into the lower thermosphere. This cyclonic vortex is always tied with:

1) Warm mesopause temperatures, inhibiting cluster ion formation.

2) Downward vertical wind components (bulk motion).

3) Low pressure near the mesopause, decreasing optical depth for solar UV radiation 


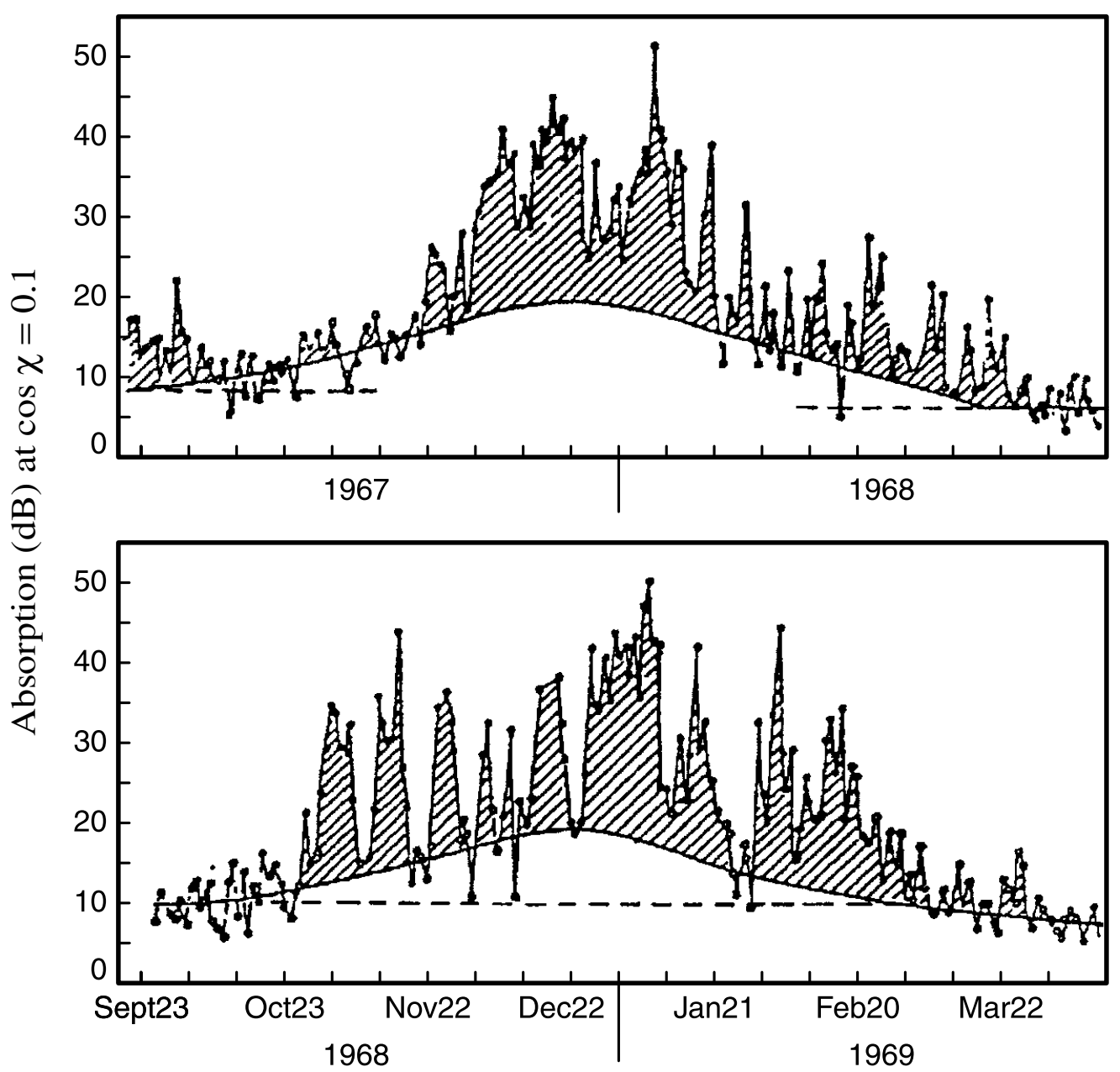

Fig. 2. Winter anomaly of ionospheric radio wave absorption in terms of the absorption at a constant zenith angle during 1967-1969. The dashed line extrapolates the trends from summer (Taubenheim, 1983).

and hence increasing the ionization rate.

All three of these conditions act in the same direction - to increase the $D$-region electron density.

In this «unitary» scenario the enhanced eddy diffusion in winter raises the average background level of $D$-region ionization and all global or local perturbations of the zonal vortex flow (for instance induced by planetary wave energy transfer from below) cause a prompt decrease or even «breakdown» of the winter anomaly in $D$-region. Both scenarios have their own merits and demerits. It seems still necessary to improve the empirical base to define coupling processes throughout the atmosphere during the winter anomaly.

The numerous experimental case studies on the stratosphere-lower ionosphere coupling in middle latitudes analyze the stratospheric temperature variations and lower ionosphere plasma density (radiowave absorption). The correlation between the temperature increment 
in the stratosphere and ionospheric absorption during the winter sudden warmings were noted for both hemispheres long ago. Figure 3 (Shapley and Beynon, 1965) demonstrates the supe-rimposed epoch analysis comparing 10 $\mathrm{mb}$ temperatures over Berlin and radiowave absorption over Lindau (Germany). The increase in absorption for the day of $10 \mathrm{mb}$ warming is evident. $D$-region electron density changes accompanying a winter stratospheric warming at $25-30 \mathrm{mb}$ in June and July for New Zealand (Christchurch, $43^{\circ} \mathrm{S}, 173^{\circ} \mathrm{E}$ ) are

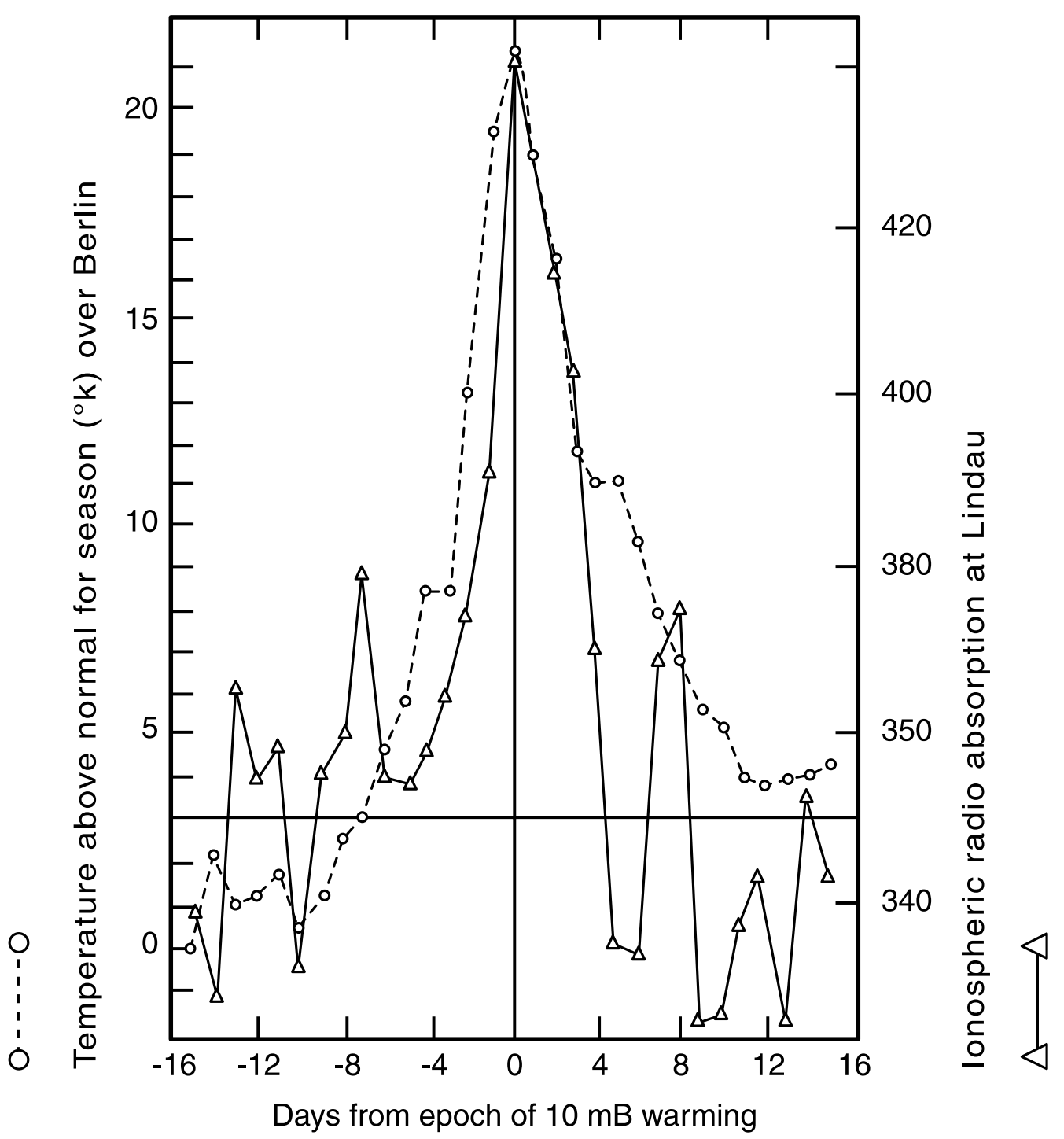

Fig. 3. Superimposed epoch analysis comparing $10 \mathrm{mbar}$ temperature over Berlin, and radiowave absorption over Lindau (Germany) (Shapley and Beynon, 1965). 
shown in fig. 4a,b (Gregory and Manson, 1975).

It is interesting that for rather low latitudes the response of absorption to the stratospheric temperature rise cannot exist for all types of warmings, but only for the strong final warmings connected with a radical change in atmospheric circulation, as demonstrated in fig. 5 for El Arenosillo, Spain $\left(37^{\circ} 6^{\prime} \mathrm{N}\right.$, $6^{\circ} 44^{\prime} \mathrm{W}$ ) (De la Morena and Kazimirovsky, 1996).

It is noteworthy that in principle we can observe the inverse correlation between both parameters, in such a way that the appearance of stratospheric warming (i.e. temperature rise at the fixed stratospheric level) coincides with a significant decrease in absorption in the $D$ region (e.g., Lastovicka, 1983). Possibly, the effect on ionospheric variability could depend on the region (Lastovicka and de la Morena, 1987), the meridional wind variability in the $D$-region (Lastovicka et al., 1990) and even on the height of stratospheric level with increasing temperature.

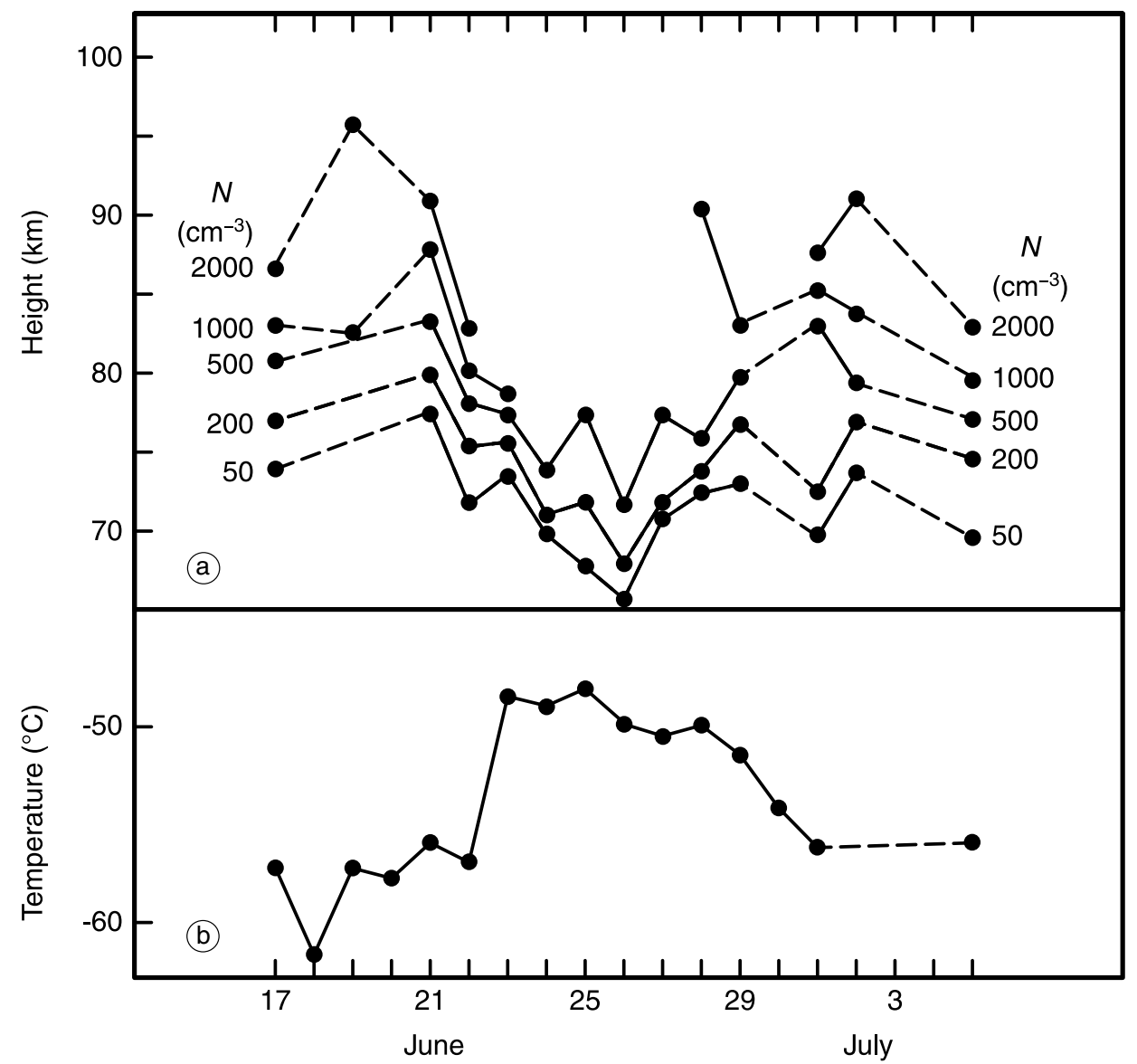

Fig. 4a,b. Ionospheric $D$-region changes accompanying a winter stratospheric warming in June and July 1963 , at Christchurch, New Zealand, $43^{\circ} \mathrm{S}, 173^{\circ} \mathrm{E}$. a) Noon electron concentration N; b) stratospheric temperature at 20-30 mbar level (about $25 \mathrm{~km}$ altitude) (Gregory and Manson, 1975). 
A3 Method. $\mathrm{f}=2830 \mathrm{KHz}$

«EL ARENOSILLO» $\left(37^{\circ} 6^{\prime} \mathrm{N} ; 6^{\circ} 44^{\prime} \mathrm{W}\right)$

$1976-1993$

KEY DAY - day of maximum stratospheric temperature on $30 \mathrm{mb}$.

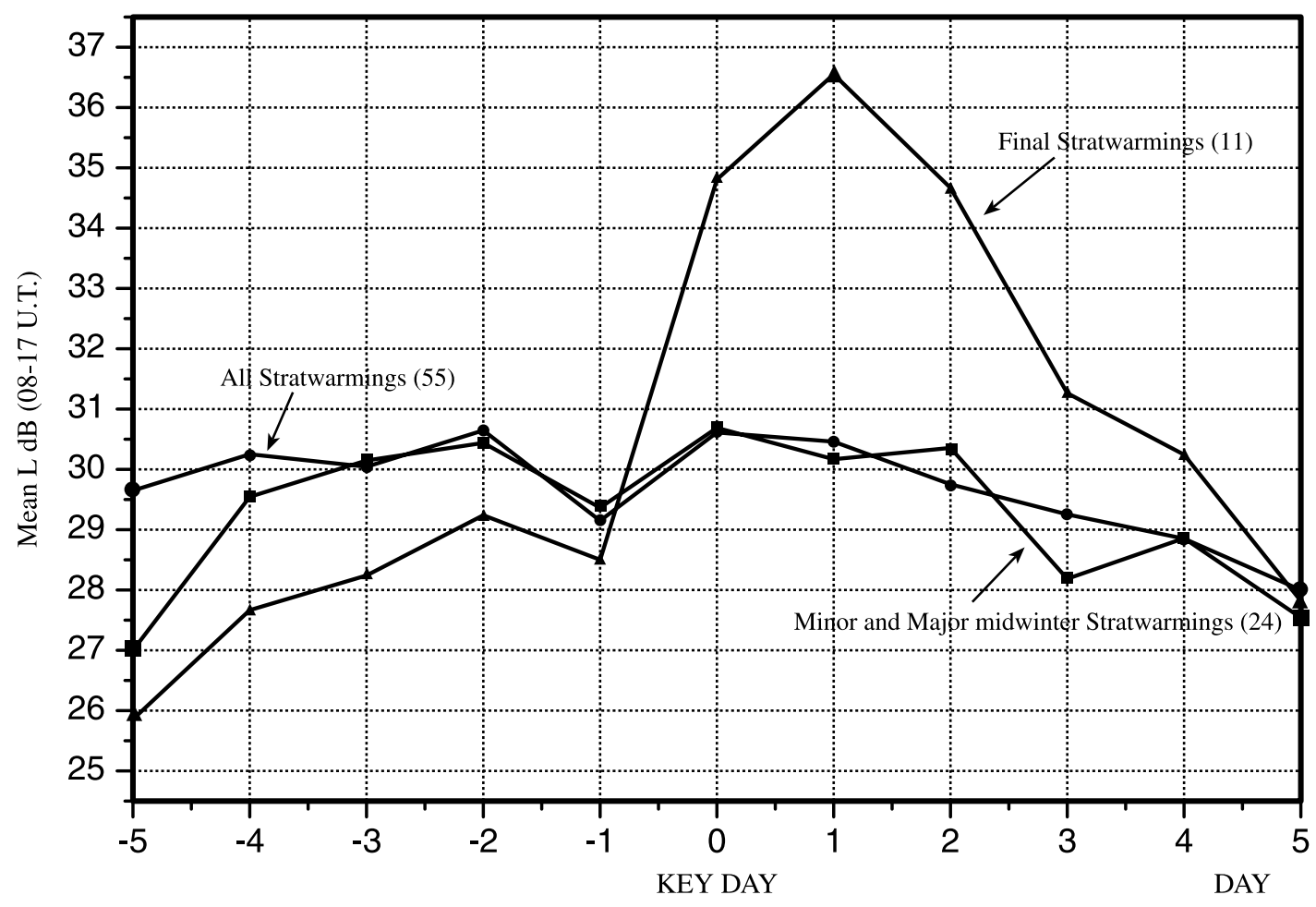

Superposed epoch method

Fig. 5. Effect of stratospheric winter warming on daytime absorption of radio waves. A3 method. Transmitted frequency $f=2830 \mathrm{kHz}, 1976-1993$, El Arenosillo, Spain $\left(37^{\circ} 06^{\prime} \mathrm{N}, 6^{\circ} 44^{\prime} \mathrm{W}\right)$. Key Day: day of maximum stratospheric temperature on 30 mbar level (de la Morena and Kazimirovsky, 1996).

The height dependence was found recently (Pietrella et al., 2001) for Antarctic, Terra Nova Bay station, located close or even inside the polar cusp region, where the geomagnetic field lines provide a direct connection between the ionosphere and magnetosphere. It means that in this area the ionospheric variability could be predominantly controlled by the interaction with the magnetosphere. So, it would be difficult to distinguish between the ionospheric variability due to meteorological effects, coming «from below» (if they exist), from the principal cause due to the influx of the energetic particles that increase the electron density and then the ionospheric absorption. Nevertheless, during the period with rather low solar activity $\left(F_{10,7}<100\right)$, we found by multiregression analysis (with statistical significance) that at the stratospheric levels between 15 and $22 \mathrm{~km}$ the influence of stratospheric temperature on ionospheric absorption seems to be more important than those due to the solar and geomagnetic activity. 
Moreover, the superimposed epoch analysis showed that the behaviour of ionospheric absorption response to the rising of the stratospheric temperature is different for the lower and upper stratosphere. On the average, for the range $13-18 \mathrm{~km}$ the ionospheric absorption increases after the stratospheric temperature maximum (so called «zero day»), but in the range $19-26 \mathrm{~km}$ the ionospheric absorption decreases after «zero day».

During the last 15-20 years, a significant number of papers have appeared with observations of regular or quasi-regular fluctuations, observed both in the neutral as well as in the ionized component of the middle atmosphere and lower ionosphere. In some of them, an attempt was made to connect the fluctuations in the $D$-region or $E$-region of the ionosphere with similar fluctuations of the meteorological parameters in the lower parts of the atmosphere. We suggest that the wellcorrelating fluctuations in the ionized and neutral component of the $D$-region, mesosphere and stratosphere are probably due to the large-scale upward propagating planetary disturbances generated in the troposphere (see numerous references in de la Morena and Kazimirovsky, 1996).

The seasonal variation in the amplitudes of quasi-periodical fluctuations of ionospheric parameters with periods of planetary waves, tides, internal gravity waves show that the amplitudes usually are maximal in winter, when conditions of upward leakage of internal waves (depending on the wind and temperature profiles between the troposphere and thermosphere) are preferable (e.g., Taubenheim, 1983). It was shown that the quasi-periodical fluctuations observed in absorption are caused not by fluctuations in the solar ionizing flux, but probably by planetary waves in the stratosphere/troposphere (e.g., Pancheva et al., 1987). Later the model of the transformation of planetary waves of tropospheric origin into waves in absorption in the lower ionosphere was developed (Lastovicka et al., 1993).

The example of the typical 7-8 and 12-13 days absorption fluctuations is presented in fig. 6 (Pancheva et al., 1991). The main features of amplitude variations are very similar at all 3 radio-frequencies (the measurements were provided by A3 method for 3 radiopaths - at Bulgaria, Czechia and Spain) for 7-8 days fluctuations while for 12-13 day fluctuations such a good similarity is observed only in December-January. $H_{1,2}$ in fig. 6 are the amplitudes of the $30 \mathrm{hPa}$ height wave 1 (full line) and 2 (dashed line) at $60^{\circ} \mathrm{N}$. The intervals of amplification of the fluctuations coincide well with the stratospheric warmings during the winter under consideration. The first «minor» warming developed with two phases: around 10 January, $1986\left(H_{2}\right.$ peak) and 20 January, $1986\left(H_{1}\right.$ peak $)$, the second «minor» warming takes place around 18 February, 1986 and the major «final» warming occurs after March 20, 1986 both as a consequence of $H_{2}$ intensification. This cold winter 1985/1986 had an enhanced tendency for the development of an elongated polar vortex, that is a pronounced planetaryscale height wave $H_{2}$ (Naujokat and Labitzke, 1993). It can be clearly seen in fig. 6 that almost each amplification of the quasi-stationary planetary waves $\mathrm{H}_{2}$ in the stratosphere leads to the activation of the 7-8 days variations in the ionospheric absorption. The behaviour of the longer-period fluctuations in absorption shows a response to the simultaneous amplification of both height waves $\left(H_{1}, H_{2}\right)$ in the stratosphere.

Therefore, we can conclude that the quasiperiodical fluctuations observed in the ionospheric absorption are really connected with the large-scale planetary disturbances generated in the lower atmosphere, which cause the stratospheric warmings.

The ionospheric dynamics certainly must be sensitive to the coupling from below. The close connection between the circulation in the lower thermosphere, mesosphere and stratosphere, e.g., nearby radar and rocket measurements demonstrate the consistent crosssections from 20 to $110 \mathrm{~km}$ (e.g., Meek and Manson, 1985).

It is generally accepted that while the International Reference Models of the zonally averaged upper mesosphere/lower thermosphere wind field are still useful for many purposes, significant discrepancies exist between them and new experimental data. The latter demonstrate 


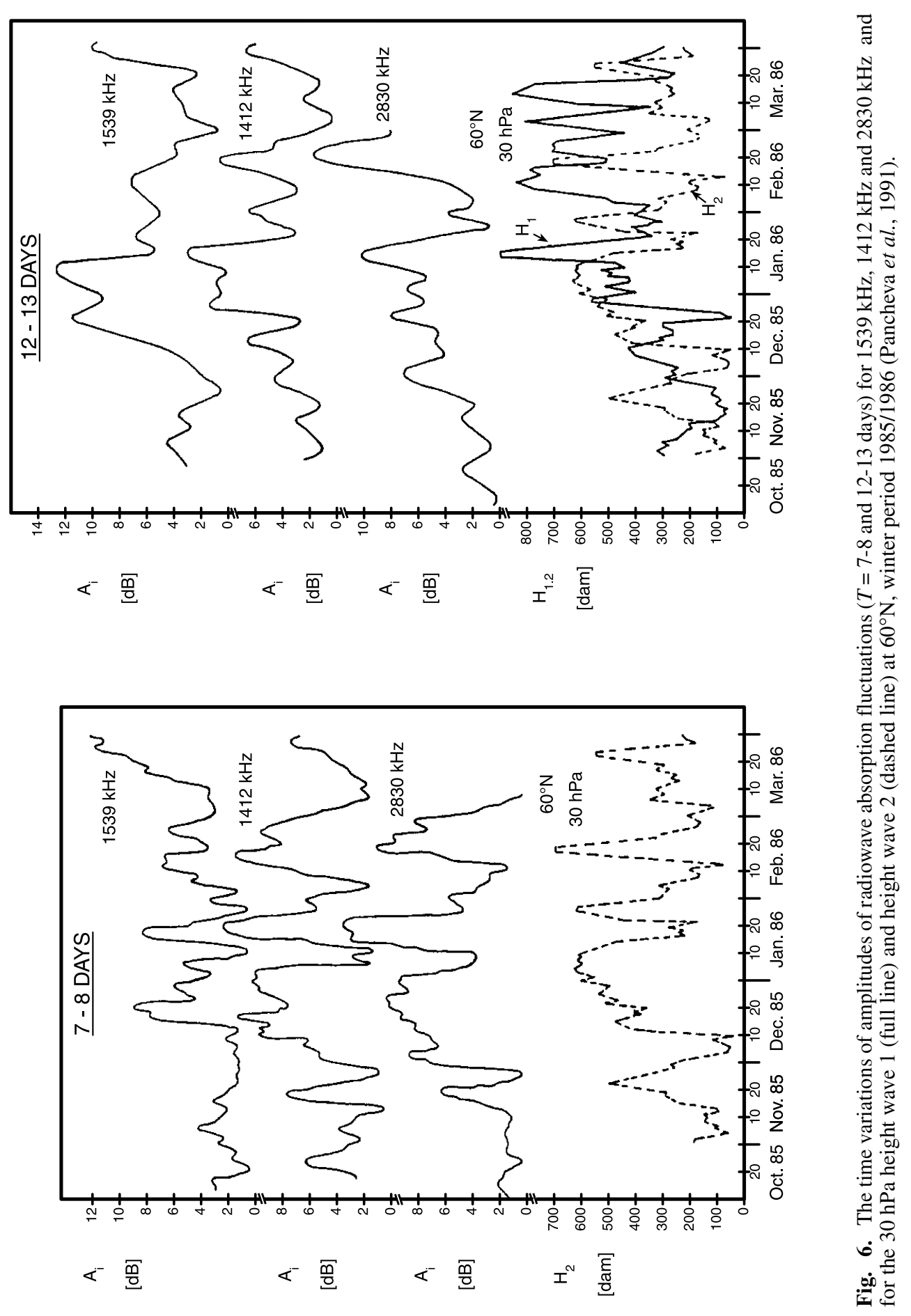


in addition to seasonal and latitudinal variations also longitude dependent variations; in addition to tides also planetary waves and intraseasonal quasiperiodical fluctuations. It is evident that sometimes the specific regional features of wind regime are very important.

If, as has been demonstrated, there is meteorological control of the lower ionosphere, we may expect the existence of a longitudinal effect on the dynamic regime due to well known longitudinal inhomogeneity of the lower atmosphere processes and due to longitudinal differences in the conditions for upward propagation of internal atmospheric waves from the lower atmosphere. The longitudinal effect has really been revealed on the basis of the simultaneous upper mesosphere/lower thermosphere wind measurements ( $D$-region) along one latitude circle at two or more sites (e.g., Kazimirovsky et al., 1988).

It has been well known since the first analyses of wind variations at the mid-latitude lower thermosphere that diurnal velocity variation could be well described as the sum of prevailing wind, diurnal wave and semidiurnal wave (e.g., Sprenger and Schminder, 1967). The existence of diurnal and semidiurnal tides at MLT-region was also demonstrated in numerous theoretical models (e.g., Forbes, 1991).

Figure 7a demonstrates prevailing wind $\left(V_{0}\right)$ monthly averaged variations for Observatory Badary (East Siberia, $52^{\circ} \mathrm{N}$ ) and Observatory Collm (Central Europe, $52^{\circ} \mathrm{N}$ ). The systematic climatological distinctions are evident, especially for the zonal circulation $\left(V_{0 \mathrm{x}}\right)$. During winter, the averaged wind over Eastern Siberia is about twice as strong as that over Central Europe. The seasonal variation of the zonal circulation depends on longitude as well: the autumn minimum over Siberia occurs earlier than that over Europe, and the spring minimum is accompanied by a reversal of wind only over Europe, but not over Siberia. The observed longitudinal effect may be partly interpreted as resulting from large scale stationary planetary waves formed at the lower thermosphere. In this case, the longitudinal variation of prevailing wind is due to the existence of such waves.

Figure $7 \mathrm{~b}$ shows that the seasonal variation of the monthly averaged semidiurnal zonal tide
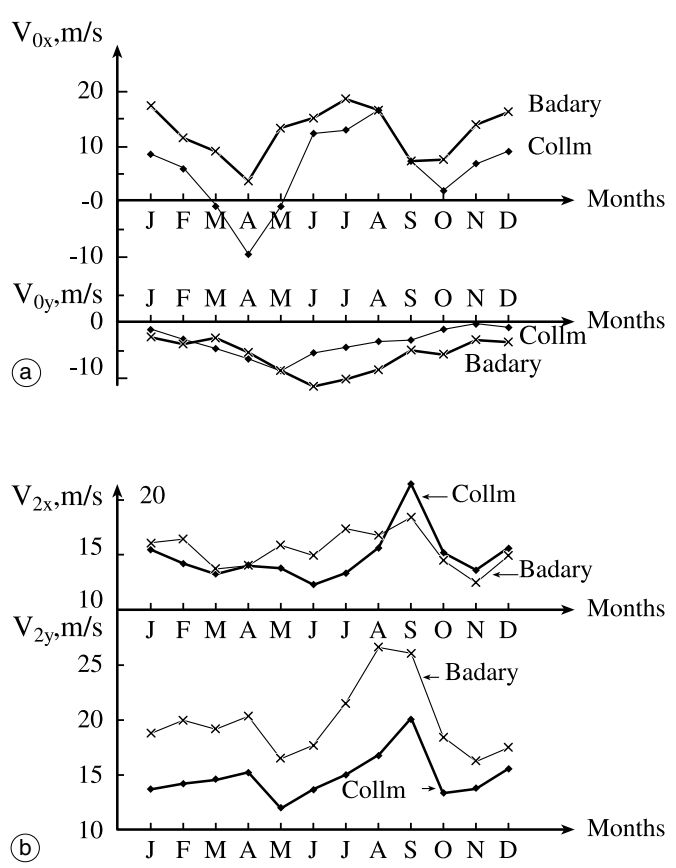

Fig. 7a,b. The non-zonality in the wind field in the lower ionosphere (Kazimirovsky et al., 1999). Observatory Badary, East Siberia, 52 ${ }^{\circ}$ N , 1975-1995. Observatory Collm, Central Europe, $52^{\circ} \mathrm{N}, 1979-$ 1997. Data are monthly averaged. $V_{\mathrm{ox}}$ - prevailing zonal wind. $V_{\text {oy }}$ - prevailing meridional wind. Positive direction - eastward and northward. $V_{2 x}$ - amplitude of the zonal semidiurnal tide. $V_{2 y}$ - amplitude of the meridional semidiurnal tide.

amplitudes $\left(V_{2 \mathrm{X}}\right)$ at both observatories are very similar with small discrepancies mainly in the summer months. The systematic climatological distinctions (buth with similar character of seasonal variations) are evident for semidiurnal meridional tide amplitudes $\left(V_{2 \mathrm{Y}}\right)$, which are systematically larger at East Siberia than at Central Europe. The longitudinal variation of semidiurnal tidal amplitude is a consequence of the longitudinal variation of zonal flow (Kazimirovsky et al., 1988, 1999).

The zonal and meridional prevailing winds at the lower thermosphere reversed westwards and southwards in a period of less than a week during stratospheric warmings. There is an 
increase in the semidiurnal tide amplitude and phase shift comparatively with undisturbed winter conditions. The response could depend on longitude as well, on the intensity and location of the warming area (e.g., Kazimirovsky et al., 1988). This response of the prevailing zonal wind for 3 observatories located on the same geographical latitude (in Canada, Central Europe and East Siberia) is demonstrated in fig. 8 .

Planetary waves contribute significantly to the variability of MLT winds. In the mesosphere

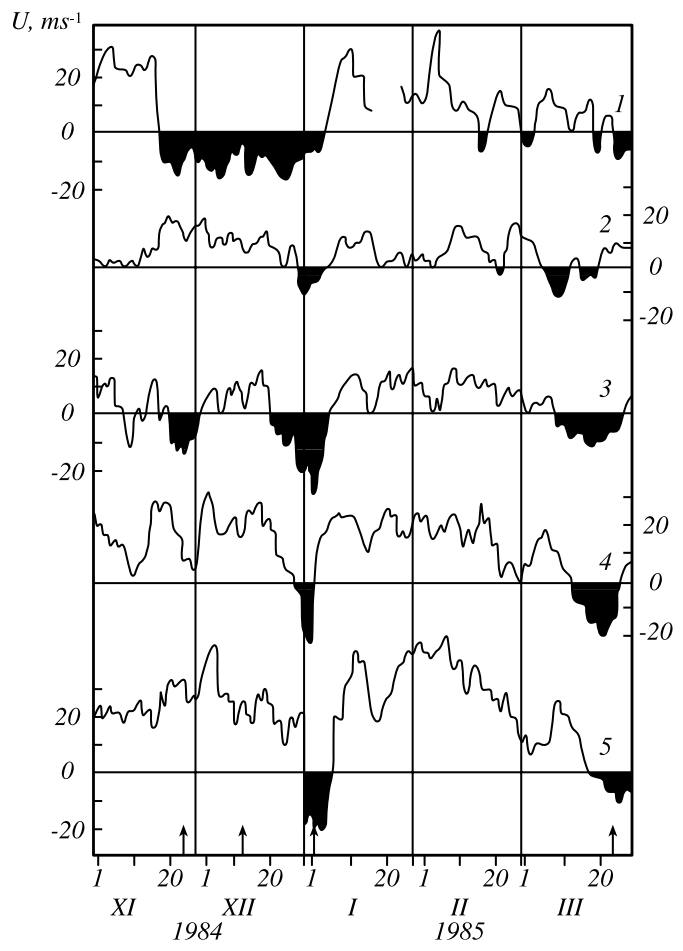

Fig. 8. The response of the prevailing zonal wind ( $\mathrm{U} \mathrm{m} / \mathrm{s}$ ) in the lower ionosphere on the stratospheric warmings during winter 1984-1985 (rather low solar activity). Positive direction - eastward. Arrows - days of stratospheric warmings (Kazimirovsky et al., 1988). 1 - Observatory Badary, East Siberia, $52^{\circ} \mathrm{N}$; 2 - Observatory Collm, Central Europe, $52^{\circ} \mathrm{N} ; 3$ Observatory Sakatoon, Canada, $52^{\circ} \mathrm{N}$, height $97 \mathrm{~km}$; 4 - Observatory Saskatoon, 89 km; 5 - Observatory Saskatoon, $80 \mathrm{~km}$. and lower thermosphere the wave fluctuations are sufficiently large to often mask the prevailing or mean state of the atmosphere. Tropospherically forced planetary waves with periods between 2 and 30 days have been observed under favourable conditions to penetrate up to heights near $110 \mathrm{~km}$. A range of wave periods has been identified but the most commonly reported periods fall into four well defined intervals which are 14-20 days, 9-12 days , 4-7 days and 1.6-2.2 days. These are often referred to as «16-day», «10day», «5-day» and «2-day» oscillations respectively, although a precise determination of the periods involved is often not possible. The observed periods vary around these values in an apparently random way possibly due to Doppler shifting by the mean winds. Thus, they form «period bands» (Lastovicka, 1997). It should be noted that waves of other periods have also been reported. It is usually assumed that these transient oscillations of the wind field in the lower thermosphere are caused by Rossby-gravity normal modes generated in the lower atmosphere (Vincent, 1990).

Who may be a preferable carrier of upward influence (especially for planetary waves) across the mesopause to the lower thermosphere and ionosphere? The atmospheric tides are the most probable candidate.The non-zonal modulation of the stratospheric and mesospheric tides penetrating to the lower thermosphere appears to generate a quasi-stationary planetary wave by demodulating the tides by energy dissipation at the lower thermosphere (e.g., Forbes, 1991). The modulation of the prevailing wind and semidiurnal tide with periods of planetary waves are shown for two midlatitude observatories (Badary and Collm) in figs. 9 and 10. The dynamic spectra were calculated for two-year interval with a data «window» of one month. Really, the spectra of fluctuations are very wide and variable and differ for different longitudes.

The general concept of significant longitudinal structure in the MLT winds was supported and developed during the international MLTCS campaigns (Manson et al., 1991), and the international DYANA Project (Singer et al., 1994; Portnyagin et al., 1994). Clear longitudinal effects were observed either for prevailing wind or semidiurnal tides. The recent cooperative 

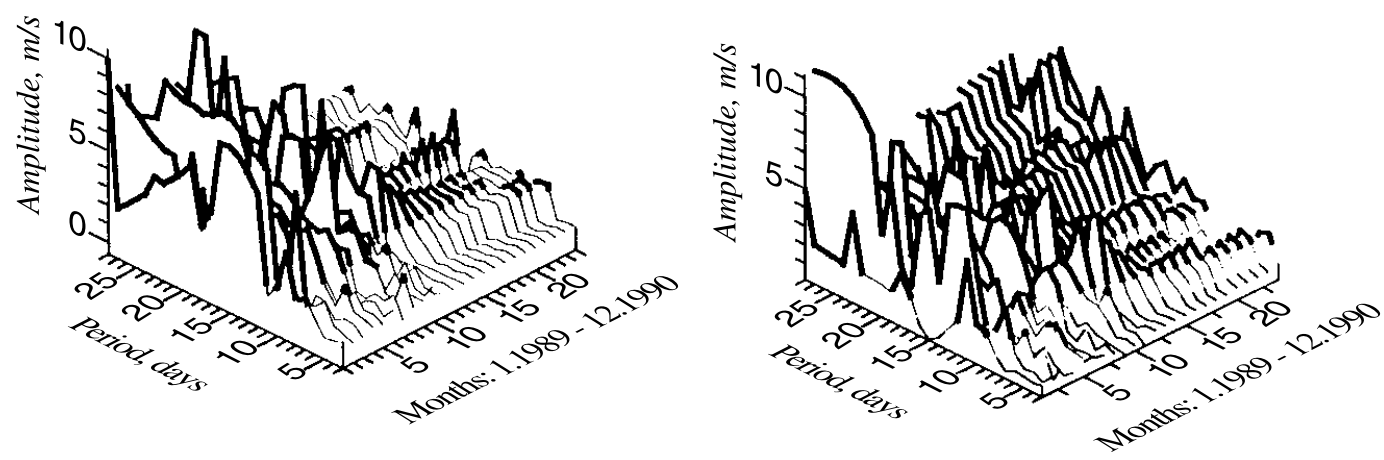

Fig. 9. Lower thermosphere zonal prevailing wind dynamic spectra at East Siberia and Central Europe.

Observatory Badary (East Siberia, 52N)

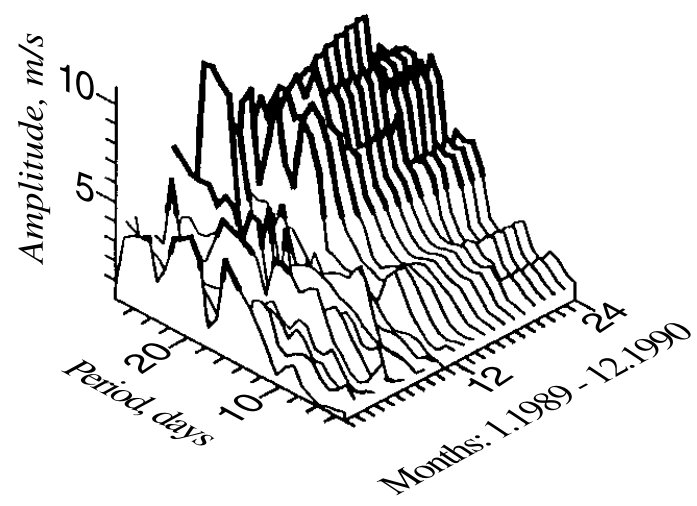

Observatory Collm (Central Europe, 52N)

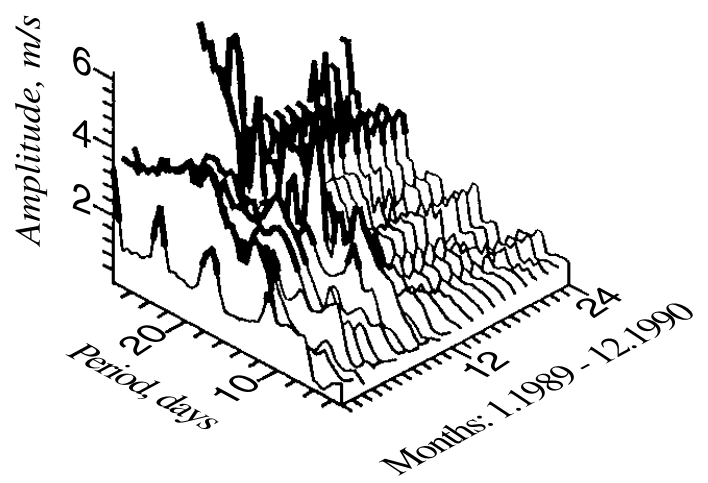

Fig. 10. Lower thermosphere zonal wind (semidiurnal tidal amplitude variations) dynamic spectra at East Siberia and Central Europe.

«CRISTA/MAHRSI Campaign» also dealt partly with upper mesosphere/lower thermosphere wind measurements and revealed some regional distinctions (e.g., Kazimirovsky et al., 1997). To determine the sources of planetary scale perturbations of the mesosphere and lower thermosphere is one of the topics in the ongoing International Program PSMOS (Planetary Scale Observing System).

Nevertheless, these processes operating in the MLT-region are still insufficiently understood.
Probably they include quasistationary planetary waves, PSW (Jacobi et al., 1999; Forbes et al., 1999), but our current understanding of the nonzonal dynamic processes contains numerous unresolved contradictions. Therefore, further experimental and theoretical investigations in this field will greatly help to extend our knowledge of the MLT-region spatial structure. Coordinated program of the simultaneous measurements of winds might be carried out at a carefully chosen series of sites comprising an east-west chain 
spanning a large sector of longitude. Such measurements in conjunction with results of the long-term zonal-mean modeling could be used to determine PSW parameters and to estimate the relative contribution of non-migrating tides. It would improve our understanding of the vertical coupling between different atmospheric regions and the coupling mechanisms between tropospheric/stratospheric non-zonal circulation systems and those which may exist in the MLTregion.

The next ionospheric region $E$, intermediate between $D$-region and $F$-region, is rather less studied in the processes of coupling from below. Nevertheless, we have some evidence concerning intensive gravity waves in the variations of the electron density and ion velocities at heights 100-140 km (e.g., Nygren et al., 1990), measured by incoherent scatter radars. The sporadic ionization in the $D$-region, so-called $E_{s}$ (sporadic $E$-layer), on the meteorologically frontal days sometimes tends to occur more frequently and with higher electron concentration than for preceding and following days.

The $E_{\text {s }}$ generation is usually connected with neutral wind shear. It is why the dynamics of the $E$-region could be crucial for $E_{s}$. The daily averaged departures of the sporadic $E$ parameters $f_{b} E_{s}$ and $h^{\prime} E_{s}$ from the corresponding monthly medians have been found to decrease during winter-time circulation disturbances connected with stratospheric warmings. The computed neutral wind shear shows decreased values during these disturbances. The effect may be due to a decrease of attenuation of AGW, due to which the vertical wavelength increases and the $E_{s}$ producing wind shear decreases with growth of vertical energy flux (numerous references in Kazimirovsky and Kokourov, 1991).

\section{Upper thermosphere/ionosphere}

This topic is rather more speculative than the lower thermosphere/ionosphere. Nevertheless, the ideas on dynamic coupling between weather at ground level and $F$-region behaviour appeared long ago (Beynon and Brown, 1951; Martyn,
1952). Of course, the attempts to forecast the surface weather from ionospheric data were too optimistic to be true. But it is evident that planetary, tidal and gravity waves launched by various sources in the troposphere and stratosphere really penetrate into the $F_{2}$ region. There are short-term correlations between meteorological and ionospheric parameters, and a number of statistically significant correlations, that may be regarded as circumstantial evidence on a longterm basis. The ionospheric response to the forcing from below should be anisotropic and subject to diurnal and seasonal variations, and should vary with geomagnetic and geographic latitude and longitude.

In fact, we know that both seasonal and daily, global and regional, composition changes at thermosphere/ionosphere heights can occur even during geomagnetically quiet periods. It is assumed that corresponding changes in the turbopause heights are responsible for the respective observations. But the turbopause heights variations could be sensitive to the meteorological dynamic influence from below. Of course, it is generally agreed that there is significant turbulence in the region $80-120 \mathrm{~km}$, although there is still some debate as to its temporal and spatial morphology. The main sources of turbulence are probably gravity waves and tides (including ones, propagating from below), and these generate turbulence by processes such as non-linear breaking, shear instabilities and critical level interactions.

We believe that planetary waves sign could be recognized in the global distribution of electron density $\left(N_{e}\right)$ in the $F_{2}$ region. The regional structure (so called continental effect) of $N_{e}$ which can be interpreted in terms of the manifestation of the climatic properties of the underlying atmosphere has been discussed on many occasions (Danilov et al., 1987). The longitude-dependent distribution of noon critical frequency $f_{0} F_{2}$ values (corresponding to maximal electron concentration) is reasonably well approximated by the sum of planetary waves with zonal wave-numbers $\mathrm{n}=1$ and $\mathrm{n}=2$. Moreover, it was shown that the seasonal variations of planetary wave amplitudes in $f_{0} F_{2}$ and in geopotential at $10 \mathrm{mb}$ level are closely correlated but with changeable time lag. 
Studies of coupling between ionospheric and stratospheric parameters sometimes yield ambiguous conclusions due to the masking effect on the ionosphere from the various geophysical factors, such as the ionizing radiation of the Sun, energetic particles fluxes, cosmic rays, etc. Thus it is desirable to make the analysis in such a manner that variations caused by these factors are removed. We proposed and successfully realized the following technique. Two stations are selected which have close geographic or geomagnetic latitudes but are well spaced in longitude so that they are likely to have substantial differences in meteorological characteristics of the lower atmosphere. For each day, the differences are determined in the ionospheric characteristics (e.g., $f_{0} F_{2}, f_{\min }$, absorption of radiowaves, etc.) between these two stations and the same differences in meteorological characteristics (e.g., stratospheric temperature, height of isobaric surface, etc.). From the comparison of the time variations of these differences, one may suppose that the effect of geophysical factors, which are almost the same for the two stations, is eliminated to a considerable extent. On the contrary, the meteorological effect due to different climatology for these two spaced locations will be stressed and thus can be identified. If meteorological factors do indeed have an influence upon the variations of ionospheric parameters, we should have a close correlation between variations of the differences described above.

The example can be demonstrated for $f_{0} F_{2}$ and stratospheric dynamics. It is well known that the winter cyclonic vortex in the stratosphere is highly dynamic and changeable. The subtropical areas of high pressure when moving northward, may deform and sometimes even split it into two independent cells. This circumstance leads to a substantial difference in the values of stratospheric pressure at two sites, located at the same latitude but at different longitudes. Does the behaviour of the $F_{2}$ ionospheric layer reflect these phenomena? Figure 11 presents the seasonal variation of daily values of differences in noon $f_{0} F_{2}\left(\Delta f_{0} F_{2}\right)$ between two sites near $55^{\circ} \mathrm{N}$ spaced in longitude by $2000 \mathrm{~km}$ and the seasonal variation of daily values of the corresponding differences in height of $30 \mathrm{hPa}$ surface $\left(\Delta h_{30}\right)$.
We can see that both during winter and equinoxes the values of $\Delta h_{30}$ and $\Delta f_{0} F_{2}$ are greater than those during summer. By the method of «superposed epochs» it was found that negative extrema of $\Delta f_{0} F_{2}$ correspond to positive extrema of $\Delta h_{30}$ and vice versa (Kazimirovsky and Kokourov, 1991).

For some time, the network of ionosonde stations in Europe had been dense enough to study the meteorological behavior of the upper ionosphere (Bibl, 1989). Even the average behaviour of the $F$-region ionization shows substantial differences with location. In Europe, the variations of the local gradients in ionization can differ by a factor of two over two locations separated by $1000 \mathrm{~km}$. This is important for understanding the meteorology of the ionosphere and for precise radio predictions.

The existence of quasi-periodic oscillations in the ionospheric $F$-region ( 2-35 days) which may be connected with planetary wave activity in the lower atmosphere is statistically evident in $f_{0} F_{2}$ variations. Figure 12 (Apostolov et al., 1998) demonstrates the averaged periodograms of $f_{0} F_{2}$ in the periodic range from $30 \mathrm{~h}$ to 40 days for the whole solar cycle 21 calculated as a mean of 126 spectra of successive 4-month intervals shifted by 1 month. The 4 stations considered lie latitudinally between $42^{\circ} \mathrm{N}$ and $60^{\circ} \mathrm{N}$. There are well expressed maxima close to 6 (4-7); 9 (7-11); 14 (11$16)$ and 29 (11-35) days. The relatively minor peaks occur near 2.5 (1.25-4) and 19 (16-21) days.

Well studied quasi-periodic oscillations with periods from 2 to 6.5 days have the following characteristics: the probability of existence and the occurrence frequency are maximum in local summer; the oscillation amplitude has maximum near equinoxes and the dominant zonal structure of the oscillations at middle latitudes is a westward traveling wave with zonal wave-number $n=1$ and $\mathrm{n}=2$. These quasi-periodic oscillations contribute significantly to the day-to-day variability of $f_{0} F_{2}$ and their contributions depend on the solar cycle, season and latitude (Altadill, 2000).

As far as the physical cause of planetary scale waves in $f_{0} F_{2}$ is concerned, it seems possible that this is the sequence of planetary waves in the neutral atmosphere (Rice and Sharp, 1977; Forbes et al., 1999), in thermospheric density. 


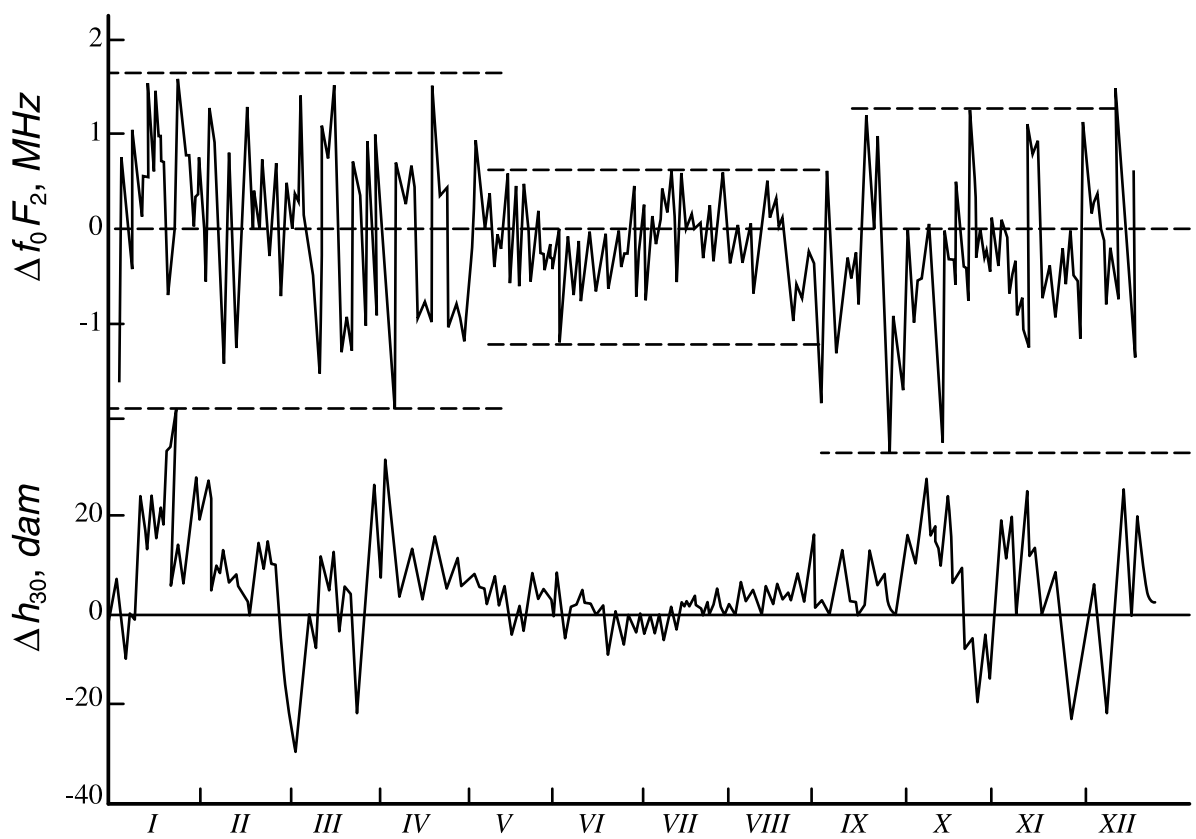

Fig. 11. Stratospheric effects on the ionospheric $F$-region. Daily values of the difference in noon values of $f_{0} F_{2}$ and in heights of an isobaric surface at $30 \mathrm{hPa}$ for two stations: Moscow (East Europe) and Ekaterinburg (Ural Mountains), located almost at 55 N. I, II, III...XII - months, 1969 (Kazimirovsky and Kokourov, 1991).

Traveling Ionospheric Disturbances (TIDs) which are the quasi-periodical spatially large-scale and medium-scale electron density perturbations possess a horizontal component of propagation velocity. It is generally accepted now that they are caused by the passage of internal gravity waves. The sources of these waves could be diverse, but they must be below the $F$-region since the wave energy (i.e. group velocity) invariably was found to be directed upwards.

From the earliest days of HF communications the presence of Travelling Ionospheric Disturbances (TIDs) and ionospheric irregularities (including $F$ spread event) were revealed and now we know that both are closely connected with internal gravity waves. At the same time, the solar cycle and seasonal variations of oscillations at different heights (e.g., Lastovicka and Mlch, 1996), the similarities in the $F_{2}$-region and lower ionosphere are such that a common source of waves in the lower and upper ionosphere of tropospheric and stratospheric origin can be postulated. In addition, planetary wave type oscillations in the $F_{2}$ region can be caused by modulation of upward propagating tides and gravity waves, so even the possibility of prediction of these oscillations from wind measurements in the lower thermosphere/ionosphere could be discussed (Lastovicka, 1997, 1998).

The sources of these waves have been identified which include severe weather disturbances, earthquakes, nuclear and industrial explosions. In the experiments provided by some investigators, for the majority of the waves the reverse group path can be followed down to the tropopause level, and comparison with meteorological data has shown that many of the possible source regions of the observed waves appear to lie in proximity to the jet stream, or close to regions of typhoons, thunderstorms, tornado, hurricanes, the convectively unstable cold polar air, subtropical heavy rainfalls, tropospheric Mesoscale 


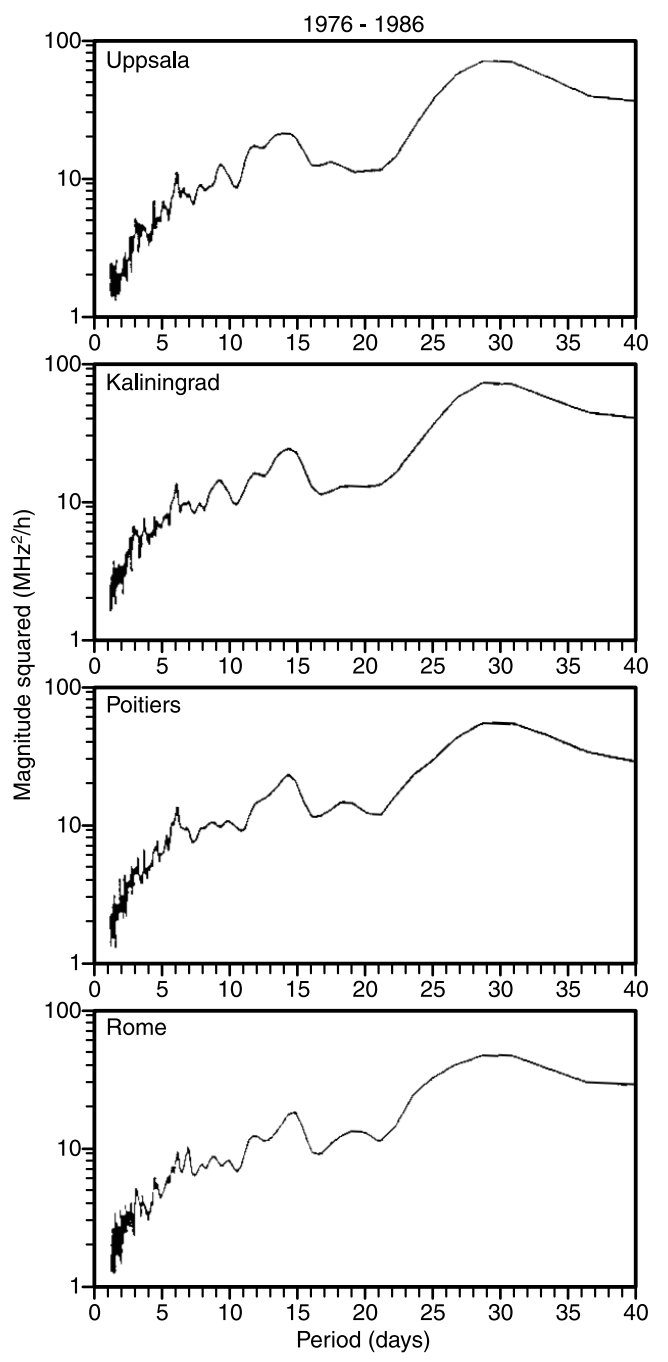

Fig. 12. Averaged periodograms of $f_{0} F_{2}$ in the periodic range from $30 \mathrm{~h}$ to 40 days for the whole solar cycle 21 , calculated as a mean of 126 spectra of successive 4-month intervals shifted by 1 month (Apostolov et al., 1998).

Convective Complexes (MCC) events, Intertropical Convergence Zone in Africa, etc. The possible generation mechanism is the non-linear interaction of shear flow instabilities in the jet stream and penetrative convection (Waldock and Jones, 1987; numerous references in Kazimirovsky and
Kokourov, 1991; Hung et al., 1991; Manzano et al., 1998; Hocke and Tsuda, 2001).

TID can be also related to tropospheric vortexes. Recently such observations were provided in China where TIDs were statistically analyzed under the base of observation of an HF Doppler array. The backward ray tracing shows that the sources of TIDs are located in the edges of Qinghai-Tibet Plateau, i.e. in the lee sides of the bulging terrain of the plateau where the vortexes are most probably produced (Wan et. al., 1998).

$F$-spread event on ionograms is often connected with wave-like structures of $F$-region irregularities induced by upward propagating AGW's of meteorological origin. It is especially true for equatorial $F$-spread. For instance, spread- $F$ occurrence in the African area was investigated by means of non great-circle radiowave propagation on the transequatorial path. The distribution of the horizontal velocity, the horizontal wavelength of the quasi-periodical structures, and the tilt of the irregularity patches led to the assumption that these structures of the equatorial $F$-spread are connected with $\mathrm{AGW}$ in the equatorial region (numerous references in Gershman et al., 1984). The small-scale ionospheric plasma events associated with severe weather-related gravity waves were also observed in situ (Kelley, 1997).

The variations of the Total Electron Content (TEC) over low-latitude stations sometimes (e.g., Shoedel et al., 1973) also show wave-like structures at the time of unusual ground pressure variations with the same period and an amplitude much greater than the amplitude of the normally observed short-period changes in TEC. The strong correlation between the oscillations of the ground pressure and the TEC gives evidence that the AGW were generated by a singular tropospheric event and propagated to ionospheric heights without significant changes in period as seen in fig. 13 for 3 African stations. Now it is impossible to deny the possibility of an association between atmospheric disturbances at ground level and in the ionosphere (Goodwin, 1980).

Recently, the effect of gravity waves was investigated successfully to estimate their contribution in the within-an-hour and hour-to- 


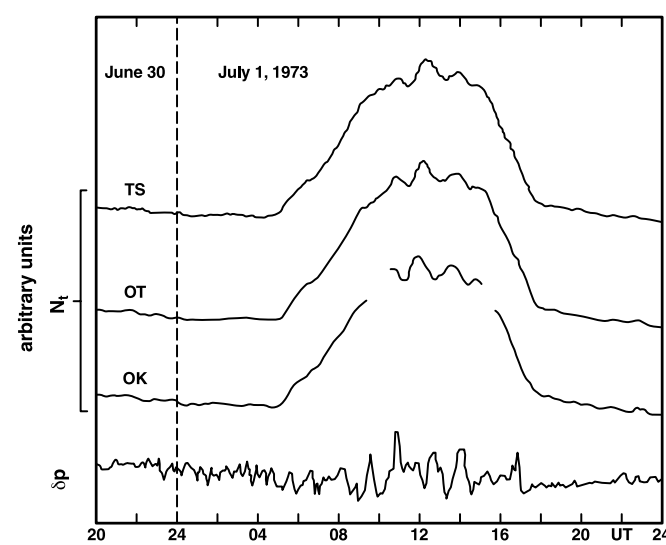

Fig. 13. Atmospheric ground pressure variation at Tsumeb (lower part), and Total Electron Content variation at Tsumeb (TS), Outjo (OT), and Okanhandja (OK) (upper part) on July 1, 1973 (Schoedel et al., 1973). hour variability of $f_{0} F_{2}$ (Boska and Lastovicka, 1996). Figure 14 (Boska et al., 1999) shows the AGW spectra for El Arenosillo, Spain, for one day. In principle, the fluctuations for electron density on the fixed heights can be caused either by fluctuations in ionized matter (i.e. gravity waves) or by fluctuations in ionizing radiation, high-energy particles related geomagnetic/ magnetospheric activity, etc. But we can believe that at middle latitudes and for rather quiet geomagnetic conditions the fluctuations in the period range $10 \mathrm{~min}-3 \mathrm{~h}$ are not of solar or magnetospheric origin.

In summary, to predict the effect of planetary and gravity waves on the variability of the ionospheric $F$-region it is necessary to know at least the scenario of indirect upward propagation of their effects. So far we have no complete modeling in this sense.

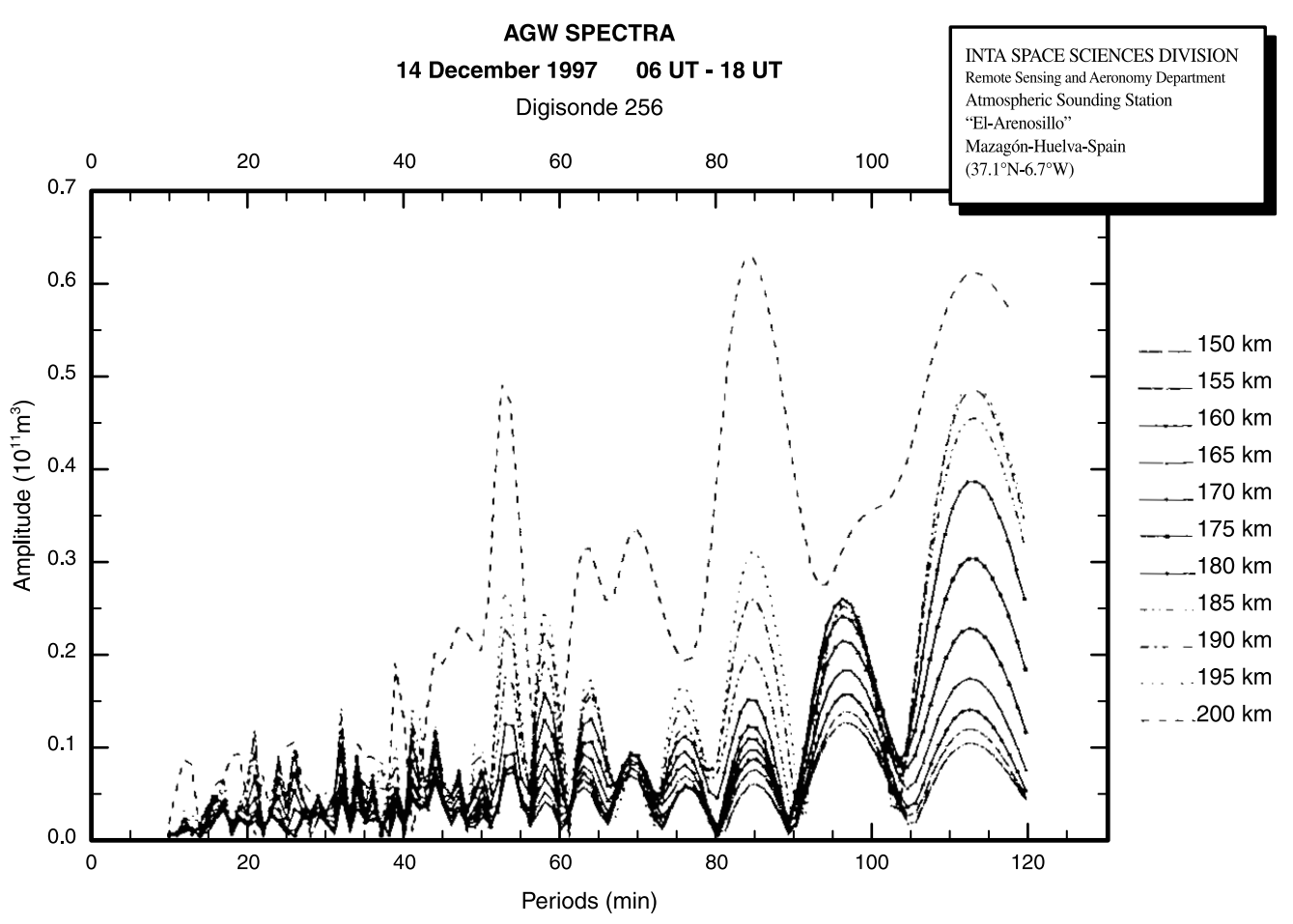

Fig. 14. Atmospheric Gravity Wave spectra in the variations of electron concentration on the fixed levels. Digisonde-256, El Arenosillo, Spain $\left(37.1^{\circ} \mathrm{N}, 6.7^{\circ} \mathrm{W}\right)$, December 14, 1997, 06UT-18 UT (Boska et al., 1999). 


\section{Thunderstorms and upper atmosphere/ ionosphere}

The widely accepted classical ideas of atmospheric electricity were:

1) Thunderstorms are the main generators of the global electric circuit, causing an electric potential between earth and the ionosphere of about 200-500 kV.

2) The influence of thunderstorms and electrical events near the ground on the atmospheric layers well above the perfect conductor («electrosphere») near $60-70 \mathrm{~km}$ is forbidden.

We know that the classical hypothesis has not yet been proved beyond doubt. Moreover, it is now widely recognized that the current systems driven by global thunderstorms and by magnetospheric plasma phenomena coexist in the middle atmosphere and above. Some recent models of thunderstorm current systems and experiments show that most of the return current from a thunderstorm generator penetrating the tropopause flows globally through the ionosphere and along plasmaspheric magnetic field lines.

The lightning generates broad electromagnetic frequency spectra and some of the wave energy propagates into the ionosphere/ magnetosphere system, where it interacts with ambient plasma particles. We have evidence that thunderstorms can be important in the global ionospheric energy budget. The thundercloud electric fields influence the $E$-region and $F$ region of the ionosphere (Suess and Tsurutani, 1998).

Recent observations have shown that intense lightning produces a number of interesting and unexpected effects in the middle and upper atmosphere above thunderstorms. Some new and diverse classes of energetic electrical effects of thunderstorms have been documented over the past 5-10 years. Two of these classes called red sprites and blue jets are large-scale optical emissions excited by lightning. Together they span the entire distance between tops of some thunderstorms and the ionosphere. These newly discovered classes of natural electric phenomena provide evidence that thunderstorms are both more energetic and capable of electrically interacting with the upper atmosphere and ionosphere to a far greater degree than has been appreciated in the past.

Sprites are very large luminous flashes that appear within the mesospheric $D$-region directly over active thunderstorm systems coincident with cloud-to-ground or intracloud lightning strokes. Triangulation of their locations and physical dimensions using simultaneous images captured from widely spaced aircrafts has shown that their terminal altitude extends to the ionosphere. The brightest region of a sprite is red and lies in the altitude range $65-75 \mathrm{~km}$. Above this there is often a faint red glow or wispy structure extending upward to about $90 \mathrm{~km}$, to the nighttime $E$ region ledge.

Jets are sporadic optical ejections, deep blue in color, that appear to erupt from the vicinity of the overshoot. Following their emergence from the tops of the thundercloud, blue jets propagate upward in narrow cones, fanning out and disappearing by about $50 \mathrm{~km}$ over a lifetime of about $300 \mathrm{~ms}$.

Theories proposed to date concerning only red sprites all involve lightning discharges acting either as a causative agent, or as a simultaneous but non-causative consequence of electrical breakdown triggered by cosmic rays. Intensive efforts, both experimental and theoretical, are underway to determine the physical mechanisms at work to produce thunderstorm $\leftrightarrow$ ionosphere effects. It is unclear whether the absorption within the ionosphere magnetosphere of the energy flowing upward from the lower atmosphere is capable of producing effects that are dynamically significant enough to qualify as a strong link between these layers. However, several longlived secondary effects within the neutral upper atmosphere may occur by way of Joule heating, photoexcitation, or electron impact excitation or ionization. Understanding where these new electrical processes fit in the solar-terrestrial system and Earth's global electrical circuit is a challenging, and inherently multidisciplinary, problem that spans traditional discipline boundaries separating the lower and upper atmosphere (Sentman and Wescott, 1995).

The evidence for direct interactions between phenomena associated with thunderstorms and 
ionosphere includes perturbations of electron density and temperature (increase of ionization at $E$-region, $E$-sporadic occurrence, increase of temperature and electron density in $F$-region), excitation of electrostatic wave turbulence and enhanced optical emissions. There are some approximate hypotheses about the physical mechanisms - e.g., upward acceleration of electrons at the moment of lightning discharge, ionization by particles, etc. (e.g., Pasko et al., 1997).

There is theoretical and experimental evidence of magnetospheric electron precipitation simulated by lightning via radio waves, particularly in the ELF-VLF range (less than 30 $\mathrm{kHz}$ ). Because the precipitating particles may be the reason for dissociation and ionization processes, it is the next channel of thunderstorm influence on the ionosphere. We have VLF signatures of ionospheric disturbances associated with «sprites» (e.g., Rycroft, 1994).

Recently, the numerical model of the interaction created in the bottom of the ionosphere above a strong horizontal (cloud-tocloud) lightning discharge was published (Cho and Rycroft, 2001). This simulation sheds light on the formation of spatially structured sprites, depending upon height, via the ratio of the ELF/ LF wave electric field (radiated by the current in the discharge) to the atmospheric neutral density.

It is interesting that the ULF-VLF electric fields were detected up to the external ionosphere over powerful Pacific Ocean typhoons (Mikhailova et al., 2000).

Thunderstorms can generate AGW as well. Reverse group ray tracing computations of AGW observed by ionospheric Doppler sounder array, show that the wave sources are in the neighborhood of storm systems. AGW at ionospheric heights are observed when severe thunderstorms are within a radius of several hundred kilometers of the ionospheric reflection points, the convective regions may be embedded in the stratiformanvils of thunderstorms. It is also known that the zones of maximal occurrence of $F$-spread over West Africa and South America coincide well with the zones of high thunderstorm activity (e.g., references in Kazimirovsky, 1983).

\section{Long-term ionosphere variations as a possible consequence of the greenhouse effect}

Releases of trace gases from human activity have a potential to cause a major change in the climate of the Earth. But there is no doubt that the subject of global climate warming due to the so called «greenhouse effect» has led to controversy, speculation and confusion. Despite the many uncertainties that remain, the consensus of most scientists knowledgeable about these matters is that global warming will occur. Some questions remain concerning the timing and the magnitude of change but there are few, if any, who dissent from this general conclusion.

The troposphere is expected to warm, the stratosphere to cool, the ozone content to decrease: the consequences of these processes on the atmosphere above $60 \mathrm{~km}$ are considered at present and overall results indicate that global change resulting from trace gas variations (e.g., $\mathrm{CO}_{2}$ and $\mathrm{CH}_{4}$ doubling) is not confined only to the lower atmosphere but also extends well into the mesosphere, thermosphere and ionosphere regions (Golytsin et al., 1996). The projected changes should also lead to some alterations in global circulation, latitudinal distributions of temperature and composition and the response of the atmospheric system to solar and auroral variability (e.g., Thomas, 1996).

Changes in a thin layer of sodium vapour, about $90 \mathrm{~km}$ above the Earth, could reveal the far-reaching effects of greenhouse gases (from 1972 to 1987 its mean height had fallen by nearly a kilometre). Although it is not possible to state with absolute certainty that the decrease in the altitude of the sodium layer is an indication of global cooling in the middle atmosphere, the behaviour of the layer clearly merits systematic surveillance in years to come (e.g., Clemesha et al., 1997).

The changes in occurrence frequency (a considerable increase) in noctilucent clouds caused either by changes in water vapour concentration or by changes in temperature, are possibly an indication of long-term anthropogenic changes. We should also consider the possible effect of anthropogenic changes in the 
mesosphere that could result from aerosols and trace gases diffusing upward into the mesosphere, where they can change the aeronomy. Polar Mesosphere Summer Echoes (Incoherent Scatter Radar) might prove to be sensitive tracers for such anthropogenic changes (e.g., Thomas, 1996).

Long-term trends in planetary wave activity at altitudes of about $80-100 \mathrm{~km}$, which are of possibly anthropogenic origin, have been extensively studied with the use of almost 30 years of absorption measurements along various radiopaths in Europe. Trends are more pronounced in daytime than night time (e.g., Lastovicka, 1997). Using different radar observations, Bremer et al. (1997) detected trends in the prevailing wind components and the amplitudes of the diurnal and semidiurnal tidal wind components in the MLT-region, at heights between about 85 and $100 \mathrm{~km}$. These trends, however, cannot be explained directly by an increasing greenhouse effect. But the well known decrease of the stratospheric ozone content may play an important role for the trends of the tidal characteristics.

According to model predictions (e.g., Rishbeth and Roble, 1992), doubling of the greenhouse gases concentration should lead to a significant cooling of the upper atmosphere and resulting changes in $F_{2}$-region parameters a decrease of the height of maximal electron concentration $\left(h_{m} F_{2}\right)$ by about $15 \mathrm{~km}$ with only small changes in $f_{0} F_{2}$. The longterm changes in $h_{m} F_{2}$ and $f_{0} F_{2}$ which were in accordance with model predictions (at least qualitatively) were reported for some ionospheric stations in the northern and southern hemispheres, but the results do not present a consistent pattern. Figure 15 shows an example of seasonal and yearly negative trends of $h_{m} F_{2}$ determined from ionosonde data of the midlatitude ionospheric station Juliusruh (Germany), 1957-1990, after

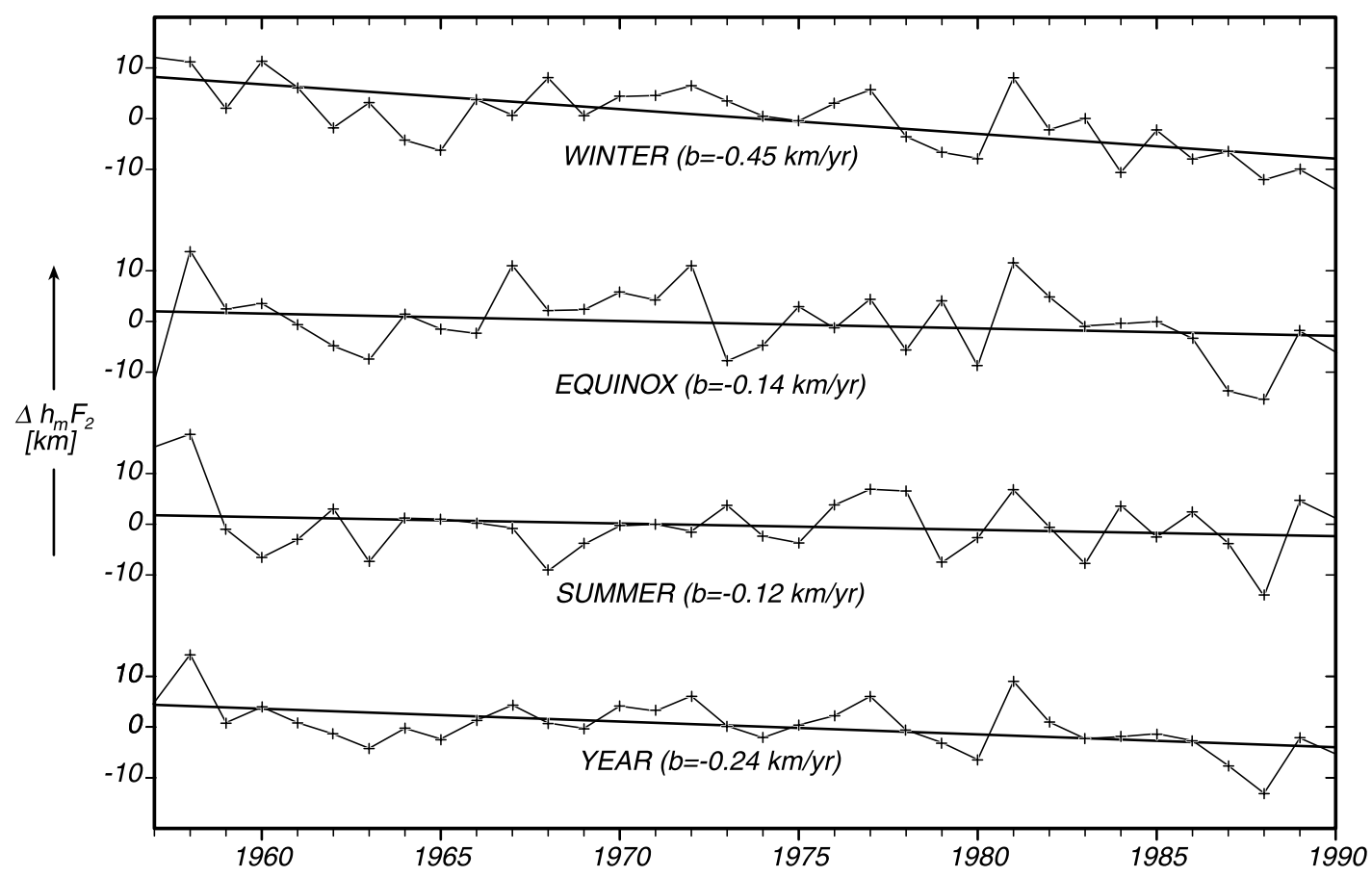

Fig. 15. Seasonal and yearly trends of $h_{m} F_{2}$ at Juliusruh (Germany) after elimination of solar and geomagnetic influences (Bremer, 1998). 
elimination of solar cycle and geomagnetic influences. Trends for winter and the whole year are statistically significant with a confidence level of more than 99\% (Bremer, 1992). Meanwhile rather recently (Marin et al., 2000) analysed the long-term trends of the $f_{0} F_{2}$ and $h_{m} F_{2}$ on Northern Hemisphere 30 ionosonde stations over the time period 1965-1991. Whereas the majority of the observed $f_{0} F_{2}$ trends were seen to be negative, the $h_{m} F_{2}$ ones were positive. The opinion of these authors that a global warming in the lower atmosphere, accompanied by cooling in the upper atmosphere, due to the greenhouse effect cannot be the cause of the detected trends. But such longterm trends could be explained by an increase in $F_{2}$-layer storm activity as a result of the increasing geomagnetic activity observed during the analysed time period. Further studies are necessary testing data homogeneity (e.g., Rishbeth, 1997; Ulich and Turunen, 1997; Bremer, 1998; Jarvis et al., 1998; Danilov, 1998).

\section{Summary}

The ionosphere, embedded in and tightly coupled to the thermosphere, is strongly influenced by couplings to other geophysical regions. In addition to ionizing energetic solar irradiation, both the magnetosphere and plasmasphere greatly affect the ionosphere by the precipitation of soft and energetic particles, by heat conduction, and by fluxes of thermal particles. Below, the middle atmosphere affects it with upwardly propagating waves (gravity waves, tides and planetary waves). Exploring these couplings effectively furthers our understanding of at least the dominant processes and interactions that play such an important role in determining the character of this part of the Earth's environment.

Perhaps the major advance in recent years has been the acceptance of meteorological processes as at least a potential cause of ionospheric variability, and not something in the realm of science fiction. Hopefully, progress will be even more rapid in the decades to come. Studies of the meteorological effects in the ionosphere are actively underway and the main aim of this review was to stimulate these investigations. Whilst some details about coupling between the middle atmosphere and ionosphere are moderately well understood, there are large gaps and deficiencies in our current understanding. Further measurements and modeling are undoubtedly required. Measurements of quantities associated with predominant neutral particles in the ionosphere are particularly important if this coupling is to be better understood. But due to the current paucity of the efficient experimental methods of measuring neutral parameters in the ionosphere, the importance of modeling studies in parallel with the new observations also must be recognized.

We must look forward to a further breakdown of the traditional isolation of the ionosphere from the lower atmosphere. Since the upper atmosphere is generally a good indicator of solar activity, one might assume that the correlation between tropospheric and ionospheric parameters may indicate such a solar-weather effect.

The question of lower/upper atmosphere coupling is a major challenge for ionospheric physics to which the growing science of aeronomy and meteorology may make an important contribution.

The only appropriate way to place these relationships on firm ground is via the study of causal mechanisms. Because of the complexity of possible relationships, it is clear that a multidisciplinary approach is required in future investigations, i.e. contributions in the fields of meteorology, climatology, aeronomy, ionospheric physics, atmospheric electricity and plasma physics. Further regional, national and international cooperative efforts are needed to organize a global monitoring system of atmospheric oscillations with various new techniques. Continuous observations from these networks permit a resolution of planetary waves, atmospheric tides and gravity waves and the hypothesis that such motions propagate upward from the lower atmosphere or are generated in situ could be examined critically with observational data.

Ionospheric weather (hour-to-hour and 
day-to-day variability of ionospheric parameters) a waits an explanation and predictions in the framework of climatological, solar cycle and seasonal variations. If we are to understand and possibly forecast ionospheric variability, then all factors, including coupling from below need to be considered.

What is desired is a quantitative appreciation of all the significant couplings, trigger mechanisms and feedback processes. Of practical concern are chains of processes that have final results important to life processes, effects on communications and technological activities, and impacts on the scientific observation of natural phenomena.

\section{Acknowledgements}

EK is grateful to Prof. Bruno Zolesi for inviting him to present the Review to the XXVI European Geophysical Society General Assembly in Nice, to Department for Geophysics and Meteorology of the University Complutense of Madrid for its hospitality during his stay in Spain (when this paper was prepared ) and to the NATO Science Program for financial support.

\section{REFERENCES}

AltadiLl, D. (2000): Planetary wave type oscillations in the ionospheric F-region, Adv. Space Res., 26, 12871296.

AndREws, D.G., J.R. Holton and C.B. LeOvy (1987): Middle Atmosphere Dynamics (monograph), Int. Geophys. Ser., 40 (Academic Press, New-York, U.S.A.).

Apostolov, E.M., D. Altadill and R. HANBABA (1998): Spectral energy contributions of quasi-periodic oscillations (2-35 days ) to the variability of $f_{0} F_{2}, A n n$. Geophys., 16, 168-188.

BEYNON, W.J.G. and G.M. BROWN (1951): Geophysical and meteorological changes in the period January-April 1949, Nature, 67, 1012-1014.

BIBL, K. (1989): Examples of meteorological behaviour of the ionosphere, AGARD Conf. Proc., 441, 36-39.

BoskA, J. and J. LAstovickA (1996): Gravity wave activity in the lower ionosphere and in the $F_{2}$ region - similarities and differences, Adv. Space Res., 18, 127-130.

Boska, J., D. Marin, G. Miro, M. Herraiz, B.A. DE LA MORENA and E.S. KAZIMIROVSKY (1999): AGW spectra in the electron density, in Proceedings $W G$ COST-251, Huelva, Spain, 215-232.
BREMER, J. (1992): Ionospheric trends in mid-latitudes as a possible indicator of the atmospheric greenhouse effect, J. Atmos. Terr. Phys., 54, 1505-1511.

BREMER, J. (1998): Trends in the ionosphere $E$ and $F$ regions over Europe, Ann. Geophys., 16, 986-996.

BREMER, J., R. SCHMINDER, K.M. GREISIGER, O. HOFFMAN, D. KuERSCHNER and W. SINGER (1997): Solar cycle dependence and long-term trends in the wind field of the mesosphere/lower thermosphere, J. Atmos. Sol.Terr. Phys., 59, 497-509.

BowHILL, S.A. (1969): Interaction between the stratosphere and ionosphere, Ann. IQSY (International Quiet Sun Year), 5, 83-95.

CHO, M. and M.J. RYCROFT (2001): Non-uniform ionization of the upper atmosphere due to the electrodynamic pulse from a horizontal lightning discharge, J. Atmos. Sol.-Terr. Phys., 63, 559-580.

Clemesha, B.R., P.P. BATISTA and D.M. SiMONICH (1997): Long-term and solar cycle changes in the atmospheric sodium layer, J. Atmos. Sol.-Terr. Phys., 59, 1673-1678.

DANILOV, A.D. (1998): Review of long-term trends in the upper mesosphere, thermosphere, and ionosphere, $A d v$. Space Res., 22, 907-915.

DANILOV, A.D., E.S. KAZIMIROVSKY, G.V. VERGASOVA and G.YA. KHACHIKJAN (1987): The Meteorological Effects in the Ionosphere (monograph), (Hydrometeorological Press, Leningrad, Russia).

DE LA MorenA, B.A. and E.S. KAZIMIROVSKy (1996): The experimental investigation of the lower ionosphere response to forcing from above and below, Scientific Report INTA 1995, El-Arenosillo, Spain.

FORBES, J.M. (1991): Middle atmosphere tides and coupling between atmospheric regions, J. Geomagn. Geoelectr., 43 (Suppl. Part 1), 597-609.

Forbes, J.M., S.E. PAlo and F.A. Marcos (1999): Longitudinal structures in lower thermosphere density, J. Geophys. Res., A104, 4773-4779.

Forbes, J.M., S.E. PALO and X. ZHIANG (2000): Variability of the ionosphere, J. Atmos. Sol.-Terr. Phys., 62, 865-893.

GAVRILOV, N.M. (1992): Internal gravity waves in the mesopause region: hydrodynamical sources and climatological patterns, Adv. Space Res., 12, 113-121.

Gershman, B.N., E.S. KaZIMIROVSKY, V.D. KoKOUROV and N.A. ChERnobrovkina (1984): F-Spread Events in the Ionosphere (monograph) (Science Press, Moscow, Russia).

Golytsin, G.S., A.I. Semenov, N.N. Shefov, L.M. FISHKOVA, E.V. LYSENKO and S.P. PEROV (1996): Longterm temperature trends in the middle and upper atmosphere, Geophys. Res. Lett., 23, 1741-1744.

Goodwin, G.L. (1980): An association between atmospheric disturbances at ground level and in the ionosphere, J. Atmos. Terr. Phys., 42, 899-906.

GREGORY, J.B. and A.H. MANSON (1975): Radiation temperatures and electron density, J. Atmos. Terr. Phys., 37, 645-653.

HARGREAVES, J.K. (1992): The Solar-Terrestrial Environment. An Introduction to Geospace - the Science of the Terrestrial upper Atmosphere, Ionosphere and Magnetosphere (monograph) (Cambridge University Press, U.K.). 
Hocke, K. and T. TSUDA (2001): Gravity waves and ionospheric irregularities over Tropical Convection Zones observed by GPS/MET radio occultation, Geophys. Res. Lett., 28, 2815-2818.

HUNG, R.J., C.C. LEE, D.L. JOHNSON and A.J. CHEN (1991): Remote sensing of mesospheric and thermospheric density perturbations induced by subtropical heavy rainfalls for spacecraft environment study, Acta Astronaut., 25, 379-393.

Jacobi, Ch., Yu.I. Portnyagin, T.V. Solovjova, P. Hoffman, W. Singer, A.N. Fahrutdinova, R.A. Ishmuratov, A.G. BeARD, N.J. Mitchell, H.G. Muller, R. Schminder, D. Kuerschner, A.H. MANSON and C.E. MEEK (1999): Climatology of the semi-diurnal tide at $52^{\circ} \mathrm{N}-56^{\circ} \mathrm{N}$ from ground-based radar wind measurements 1985-1995, J. Atmos. Sol.Terr. Phys., 61, 975-991.

JARVIS, M.J., B. JENKINS and G.A. RoDGERs (1998): Southern Hemisphere observations of long-term decrease in $F$-region altitude and thermospheric wind providing possible evidence for global thermospheric cooling, J. Geophys. Res., A103, 20,774-20,787.

JoHnson, R.M. and T.L. KILLEEN (Editors) (1995): The upper mesosphere and lower thermosphere: a review of experiment and theory, Geophys. Monogr. Am. Geophys. Union, 87.

KAZIMIROVSKY, E.S. (1983): Thunderstorm effects on the ionospheric processes - A review, Res. Geomagn. Aeron. Solar Phys. (Science Press, Moscow, Russia), 66, 170-192.

KAZIMIROVSKY, E.S. and V.D. KoKOUROV (1991): The tropospheric and stratospheric effects in the ionosphere, J. Geomagn. Geoelectr., 43 (Suppl. Part 1), 551-562.

KAZIMIROVSKY, E.S.,A.H. MANSON and A.H. MEEK (1988): Winds and waves in the middle atmosphere at Saskatoon, Collm and Badary, J. Atmos. Terr. Phys., 50, 243-250.

KAZIMIROVSKY, E.S., V.D. KoKOUROV and G.V. VERGASOVA (1997): The dynamical structure of the lower thermosphere during the CRISTA/MAHRSI campaign, Adv. Space Res., 20, 1233-1236.

KAZIMIROVSKY, E.S., O.M. PIROG and G.V. Vergasova (1999): The upper mesosphere/lower thermosphere wind field nonzonality as possible sign of the external forcing from above and below, Adv. Space Res., 24, 649-655.

KELLEY, M. (1997): In situ ionospheric observations of severe weather-related gravity waves and associated small-scale plasma events, J. Geophys. Res., A102, 329-335.

KRASSOVSKY, V.I. (1977): Internal gravity waves near the mesopause and the hydroxil emission, Ann. Geophys., 33, 347-352.

LASTOVICKA, J. (1983): Winter anomaly studies in Czechoslovakia (a review), Bulg. Geophys. J., 9, 17-26.

LASTOVICKA, J. (1997): Long-term trends in the upper middle atmosphere as detected by ionospheric measurements, Adv. Space Res., 20, 2065-2074.

LASTOVICKA, J. (1998): Can planetary wave type oscillations in the $F_{2}$ region be caused by modulation of upward propagating tides and be predicted from wind measurements in the lower thermosphere?, in Proceedings 2nd COST-251 Workshop, Rutherford Appleton Lab., Didcot, U.K., 234-238.

LAstovicKA, J. and B.A. DE LA MORENA (1987): The response of the lower ionosphere in Central and Southern Europe to the anomalous stratospheric conditions, Phys. Scr., 35, 902-905.

LASTOVICKA, J. and P. MLCH (1996): Solar cycle effect on oscillations in the period range of 2-20 days in the $F$ region of the ionosphere, Ann. Geofis., 39 (4), 783-790.

LASTOVICKA, J., D. PANCHEVA and X.-J. ZHANG (1990): On some differences in the behaviour of the upper and lower part of the lower ionosphere in relation to meteorological parameters, Ann. Geophys., 8, 599-608.

LASTOVICKA, J., A. EBEL and A. ONDRASKOVA (1993): On the transformation of planetary waves of tropospheric origin into waves in radio wave absorption in the lower ionosphere, Studia Geophys. Geodet., 38, 71-81.

Manson, A.H., C.E. MeEK, R. SCHMinder, D. Kuerschner, R.R. Clark, H.G. Mueller, R.A. VinCENT, A. Phillips, C.J. Fraser, W. Singer and E.S. KAZIMIROVSKY (1990): Tidal winds from the MLT global radar network during the first LTCS campaign September 1987, J. Atmos. Terr. Phys., 52, 175-187.

Manson, A.H. C.E. MeEK, S.K. AVERY, R.A. Fraser, R. SCHMINDER, D. KUERSCHNER and E.S. KAZIMIROVSKY (1991): Tidal winds from the mesosphere, lower thermosphere global radar network during the second LTCS campaign - December 1988, J. Geophys. Res. A96, 1117-1126.

Manzano, J.R., S.M. RadiCElla, M.M. Zossi DE ARTIGAS, A.N. FILIPPI DE MANZANO and A.H. COSIO DE RAgOnE (1998): Troposphere-ionosphere interaction during tropospheric Mesoscale Convective Complexes (MMC) events, J. Atmos. Sol.-Terr. Phys., 60, 585-594

Marin, D., A.V. Mikhailov, B.A. DE LA Morena and M. HERRAIZ (2000): Tendencias a largo plaza en region $F_{2}$ de la ionosfera y su relacion co la actividad magnetica, Fisica de la Tierra, Universidad Complutense de Madrid, Spain, 12, 263-280.

MARTYN, D.F. (1952): Troposphere-ionosphere relationships, Geophys. Res. Pap., US.A., 12, 31-33.

MeEK, C.E. and A.H. MANson (1985): Comparison of Primrose Lake $\left(54^{\circ} \mathrm{N}, 110^{\circ} \mathrm{W}\right)$ Rocket winds $(20-60$ $\mathrm{km})$ and Saskatoon $\left(52^{\circ} \mathrm{N}, 107^{\circ} \mathrm{W}\right) \mathrm{MF}$ radar winds (60-110 km), J. Atmos. Terr. Phys., 47, 477-487.

MeteorologicAl GlosSARY (1991): HMSO, Meteorological Office, London,U.K.

MikHaiLOV, A.V. (2000): Geomagnetic control of the $f_{0} F_{2}$ trends, Ann. Geophys., 18, 653-665.

MikHailova, G.A., Yu.M. MikHailov and O.V. KAPUSTINA (2000): ULF-VLF electric fields in the external ionosphere over powerful typhoons in Pacific Ocean, Int. J. Geomagn. Aeron., 2 (2) (in Internet).

NAUjOKAT, B. and K. LABITZKE (1993): Collection of Reports on the Stratospheric Circulation During the Winters 1974/1975 - 1991/1992, SCOSTEP Secretariat, University of Illinois, U.S.A.

NyGREN, T., T. TURUNEN and H. Rishbeth (1990): Interference of tidal and gravity waves in the iono- 
sphere and an associated sporadic E-layer, J. Atmos. Terr. Phys., 52, 609-624.

OFFERMAN, D., H. BRUKELMAN, J. BARNETT, K. LABITZKE, K. TORKAR and H. WIDDEL (1982): A scale analysis of the $D$-region winter anomaly, J. Geophys. Res., 87 , 8286-8306.

PANChEVA, D., E. APOSTOLOV, J. LASTOVICKA and J. BosKA (1987): Long-period fluctuations observed in the ionospheric absorption variations, Studia Geophys. Geod., 31, 301-306.

PANCheVA, D., J. LASTOVICKA and B.A. DE LA MorenA (1991): Quasi-periodic fluctuations of ionospheric absorption in relation to planetary activity in the stratosphere, J. Atmos. Terr. Phys., 53, 1151-1155.

PASKO, V.P., U.S. INAN, T.F. BELL and Y.N. TARANENKO (1997): Sprites produced by quasi-electrostatic heating and ionization in the lower ionosphere, J. Geophys. Res., A102, 4529-4561.

Pietrella, M., E.S. KaZimirovsky, G. DE Franceschi, P. GRIGIONI and C. SCOTTO (2002): Could we find any signal of the stratosphere-ionosphere coupling in Antarctic?, Ann. Geophys., 45 (1), 145-153 (this volume).

Portnyagin, Yu.I., N.A. MaKarov, R.P. Chebotarev, A.N. NIKONOV, E.S. KAZIMIROVSKY, V.D. KOKOUROV, V.V. Sidorov, A.N. FAHRUtDinOVA, G. CEVOlani, R.R. Clark, D. Kuerschner, R. SCHMINDER, A.H. MANSON, C.E. MEEK, H.G. Muller, J.C. STOdDARD, W. SINGER and P. HOFFMANN (1994): The wind regime of the mesosphere and lower thermosphere during the DYANA campaign - II. Semidiurnal tide, J. Atmos. Terr. Phys., 56, 1731-1740.

RICE, C.J. and L.R. SHARP (1977): Neutral atmospheric waves in the thermosphere and troposphere weather systems, Geophys. Res. Lett., 4, 315-317.

RISHBETH, H. (1997): Long-term changes in the ionosphere, Adv. Space Res., 20, 2149-2156.

RishBETH, H. and R.G. RoBLE (1992): Cooling of the upper atmosphere by enhanced greenhouse gases - Modeling of thermospheric and ionospheric effects, Planet. Space Sci., 40, 1011-1026.

RYCROFT, M.J. (1994): Some effects in the middle atmosphere due to lightning, J. Atmos. Terr. Phys., 56, 343-348.

SCHWENTEK, H. (1976): Manual on Ionospheric Absorption Measurements, World Data Center A, U.S.A., Chapter 6.

SCHOEdEl, J.P., J. KLOSTERMEYER, J. ROETTGER and G. STILKE (1973): Evidence for tropospheric-ionospheric coupling by atmospheric gravity waves, Z. Geophys., 39, 1063-1066.
SEntMan, D.D. and E.M. WescotT (1995): Red sprites and blue jets: Thunderstorm excited optical emissions in the stratosphere, mesosphere, and ionosphere, Phys. Plasmas, 2, 2415-2419.

SHAPLEY, A.H. and W.J.G. BEYNON (1965): Winter anomaly in the ionospheric absorption and stratospheric warmings, Nature, 206, 1242-1243.

Singer, W., P. Hoffman, A.H. Manson, C.E. MeEK, R. SCHMINDER, D. KUERSCHNER, G.A. KOKIN, A.K. KNYAZEV, YU.I. POTNYAGIN, N.A. MAKAROV, A.N FAHRUTDinova, V.V. SidOROV, G. CEVOLANI, H.G. MulLER, E.S. KAZIMIROVSKY, V.A. GAIDUKOV, R.R. CLARK, R.P. CHEBOTAREV and Y. KARADJAEV (1994): The wind regime of the mesosphere and lower thermosphere during DYANA campaign - I. Prevailing wind, J. Atmos. Terr. Phys., 56, 1717-1730.

SOLAR-TERRESTRIAL ENERGY PROGRAM (STEP) 19901997 (1990): SCOSTEP Secretariat, University of Illinois, Urbana, U.S.A.

Solomon, S.C. (2000): Modeling of the thermosphere/ ionosphere system, Phys. Chem. Earth (C), 25, 499- 503.

SPRENGER, K. and R. SCHMinder (1967): On the significance of ionospheric drift measurements in the LF-range, J. Atmos. Terr. Phys., 29, 183-199.

SuESS, S.T. and B.T. TsuRUTANI (Editors) (1998): From the Sun Auroras, Magnetic Storms, Solar Flares, Cosmic Rays (monograph), Am. Geophys. Union, Washington, D.C., U.S.A.

TAubenheim, J. (1983): Meteorological control of the $D$ region, Space Sci. Res., 34, 397-411.

THomAs, G.E. (1996): Global change in the mesospherelower thermosphere region: has it already arrived?, $J$ Atmos. Terr. Phys., 58, 1629-1656.

ULICH, T. and E. TURUNEN (1997): Evidence for long-term cooling of the upper atmosphere in ionosonde data, Geophys. Res. Lett., 24, 1103-1107.

VINCENT, R.A. (1990): Planetary and gravity waves in the mesosphere and lower thermosphere, Adv. Space Res., 10, 93-101.

WALDOCK, J.A. and J.B. JONES (1987): Source regions of medium traveling ionospheric disturbances observed at mid-latitudes, J. Atmos. Terr. Phys., 49, 105-114.

WAN, W., H. YUAN, B. NING, J. LIANG and F. DING (1998): Traveling ionospheric disturbances associated with tropospheric vortexes around Qinghai-Tibet Plateaux, Geophys. Res. Lett., 26, 3775-3778.

WhitTEN, R.C. and I.G.PopPofF (1971): Fundamentals of Aeronomy (monograph) (John Wiley, New York, U.S.A.).

WICKWAR, V.B. and H.C. CARLSON (1999): Ionospheric and thermospheric couplings: vertical, latitudinal and longitudinal, J. Atmos. Sol.-Terr. Phys., 61, 141-152. 